\title{
Comprehensive registry of esophageal cancer in Japan, 2014
}

\author{
Masayuki Watanabe ${ }^{1}$ (1) $\cdot$ Yasushi Toh $^{2} \cdot$ Ryu Ishihara $^{3} \cdot \mathrm{Koji} \mathrm{Kono}^{4} \cdot$ Hisahiro Matsubara $^{5} \cdot$ Kentaro Murakami $^{5}$. \\ Kei Muro ${ }^{6} \cdot$ Hodaka Numasaki $^{7}$. Tsuneo Oyama ${ }^{8} \cdot$ Soji Ozawa $^{9} \cdot$ Hiroshi Saeki $^{10} \cdot$ Koji Tanaka $^{11} \cdot$ Takahiro Tsushima $^{12}$. \\ Masaki Ueno ${ }^{13} \cdot$ Takashi Uno $^{14} \cdot$ Toshiyuki Yoshio $^{15}$. Shiyori Usune ${ }^{16} \cdot$ Arata Takahashi $^{16} \cdot$ Hiroaki Miyata $^{16} \cdot$ The \\ Registration Committee for Esophageal Cancer of the Japan Esophageal Society
}

Received: 14 July 2021 / Accepted: 6 September 2021 / Published online: 22 September 2021

(c) The Author(s) 2021

\begin{abstract}
Background The registration committee for esophageal cancer in the Japan Esophageal Society (JES) has collected the patients' characteristics, treatment, and outcomes annually.

Methods We analyzed the data of patients who had visited the participating hospitals in 2014. We collected the data with a webbased data collection system using the National Clinical Database. We used the Japanese Classification of Esophageal Cancer 10th edition by JES and the TNM classification 7th edition by the Union of International Cancer Control (UICC) for cancer staging. Results A total of 9026 cases were registered from 344 institutions in Japan. Squamous cell carcinoma and adenocarcinoma accounted for $87.9 \%$ and $7.1 \%$, respectively. The 5-year survival rates of patients treated using endoscopic resection, concurrent chemoradiotherapy, radiotherapy alone, and esophagectomy were $87.1 \%, 33.7 \%, 25.3 \%$, and $59.3 \%$, respectively. Esophagectomy was performed in 5204 cases. Concerning the approach used for esophagectomy, $48.1 \%$ of the cases were treated thoracoscopically. The operative mortality (within 30 days after surgery) was $0.75 \%$, and the hospital mortality was $2.0 \%$. The survival curves showed an excellent discriminatory ability both in the clinical and pathologic stages by the JES system. The survival of pStage IV was better than IIIC in the UICC system, because pStage IV included the patients with supraclavicular lymph-node metastasis (M1 LYM).
\end{abstract}

Conclusion We hope that this report contributes to improving all aspects of diagnosing and treating esophageal cancer in Japan.

Keywords Esophageal cancer $\cdot$ Esophagectomy $\cdot$ Radiotherapy $\cdot$ Chemotherapy $\cdot$ Endoscopic resection $\cdot$

Chemoradiotherapy

\section{Preface 2014}

We sincerely appreciate the outstanding contributions of many physicians in the registry of esophageal cancer cases. The Comprehensive Registry of Esophageal Cancer in Japan, 2014 was published here. Since 2019, the data collection method was changed from an electronic submission to a web-based data collection using the National Clinical Database (NCD).

These data were first made available on July 15, 2021, as the Comprehensive Registry of Esophageal Cancer in Japan, 2014.

The authors were members of the Registration Committee for Esophageal Cancer, the Japan Esophageal Society, and made great contribution to the preparation of this material.

Masayuki Watanabe

masayuki.watanabe@jfcr.or.jp

Extended author information available on the last page of the article
Personal information was replaced with individual management code inside each institute, and the NCD collected only anonymized information. The registry complies with the Act for the Protection of Personal Information.

We briefly summarized the Comprehensive Registry of Esophageal Cancer in Japan, 2014. According to the subject year, the Japanese Classification of Esophageal Cancer 10th by the Japan Esophageal Society (JES) [1] and the Union of International Cancer Control (UICC) TNM Classification 7 th [2] were used for cancer staging. A total of 9026 cases were registered from 344 institutions in Japan. Tumor locations were cervical in $4.8 \%$, upper thoracic in $12.9 \%$, middle thoracic in $46.5 \%$, lower thoracic in $27.2 \%$, and esophagogastric junction in 7.8\%. Superficial carcinomas (Tis, $\mathrm{T} 1 \mathrm{a}, \mathrm{T} 1 \mathrm{~b}$ ) were $37.2 \%$. As for the histologic type of biopsy specimens, squamous cell carcinoma and adenocarcinoma accounted for $87.9 \%$ and $7.1 \%$, respectively. Regarding clinical results, the 5-year survival rates of patients treated 
using endoscopic resection, concurrent chemoradiotherapy, radiotherapy alone, and esophagectomy were $87.1 \%, 33.7 \%$, $25.3 \%$, and $59.3 \%$, respectively. The endoscopic submucosal dissection accounted for $92.6 \%$ of endoscopic resection. Esophagectomy was performed in 5204 cases. Concerning the approach used for esophagectomy, $48.1 \%$ of the cases were treated thoracoscopically. The operative mortality (within 30 days after surgery) was $0.75 \%$, and the hospital mortality was $2.0 \%$. The Kaplan-Meier survival curves diverged according to the $\mathrm{N}$-grade both in the JES and the UICC classifications. The survival curves showed an excellent discriminatory ability both in the clinical and pathologic stages by the JES system. In contrast, in the UICC system, the survival of cStage IIB was better than those of IB and IIA, while the survival curves were almost identical between cStage IIIc and IV. Also, the survival curve of pStage IIB was better than that of IIA, and the survival of pStage IV was better than that of IIIC. pStage IV in the UICC system included the patients with supraclavicular lymph-node metastasis (M1 LYM), which is probably the reason for the better prognosis of pStage IV than pStage IIIC.

We hope that this Comprehensive Registry of Esophageal Cancer in Japan 2014 will help to improve all aspects of the diagnosis and treatment of esophageal cancer in Japan.

\section{Contents}

\section{Clinical factors of esophageal cancer patients treated in 2014}

\section{Institution-registered cases in 2014}

2. Patient background

Table 1 Age and gender

Table 2 Performed treatment

Table 3 Tumor location

Table 4 Histologic types of biopsy specimens

Table 5 Depth of tumor invasion, cT (UICC TNM 7th)

Table 6 Lymph-node metastasis, cN (UICC TNM 7th)

Table 7 Distant metastasis, cM (UICC TNM 7th)

Table 8 Clinical stage (UICC TNM 7th)

\section{Results of endoscopically treated patients in 2014}

Table 9 Details of endoscopic treatment for curative intent

Table 10 Complications of EMR/ESD

Table 11 Pathologic depth of tumor invasion of EMR/ ESD specimens

Figure 1 Survival of patients treated with EMR/ESD
Figure 2 Survival of patients treated with EM/ESD according to the pathological depth of tumor invasion, pT (JES 10th)

Figure 3 Survival of patients treated with EMR/ESD according to the lymphatic and venous invasion

\section{Results in patients treated with chemotherapy and/or radiotherapy in 2014}

Table 12 Dose of irradiation (non-surgically treated cases)

Table 13 Dose of irradiation (surgically treated cases) Figure 4 Survival of patients treated

with chemotherapy and/or radiotherapy

Figure 5 Survival of patients treated with definitive chemoradiotherapy according to the clinical stage (UICC TNM 7th)

Figure 6 Survival of patients underwent radiotherapy alone according to the clinical stage ( UICC TNM 7th)

\section{Results in patients who underwent esophagectomy in 2014}

Table 14 Treatment modalities of esophagectomy

Table 15 Tumor location

Table 16 Approaches to tumor resection

Table 17 Video-assisted surgery

Table 18 Fields of lymph-node dissection according to the location of the tumor

Table 19 Reconstruction route

Table 20 Organs used for reconstruction

Table 21 Histological classification

Table 22 Depth of tumor invasion, pT (JES 10th)

Table 23 Pathological grading of lymph-node

metastasis, pN (JES 10th)

Table 24 Pathological findings of lymph-node

metastasis, pN (UICC TNM 7th)

Table 25 Pathological findings of distant organ metastasis, pM (JES 10th)

Table 26 Residual tumor

Figure 7 Survival of patients who underwent

esophagectomy

Figure 8 Survival of patients who underwent

esophagectomy according to the clinical stage (JES

10th)

Figure 9 Survival of patients who underwent esophagectomy according to the clinical stage (UICC TNM

7th)

Figure 10 Survival of patients who underwent esophagectomy according to the depth of tumor invasion, pT (JES 10th) 
Figure 11 Survival of patients who underwent esophagectomy according to lymph node metastasis (JES 10th)

Figure 12 Survival of patients who underwent esophagectomy according to lymph node metastasis (UICC TNM 7th)

Figure 13 Survival of patients who underwent esophagectomy according to the pathological stage (JES 10th)

Figure 14 Survival of patients who underwent esophagectomy according to the pathological stage (UICC TNM 7th)

Figure 15 Survival of patients who underwent esophagectomy according to the residual tumor (R)

\section{Clinical features of esophageal cancer patients treated in 2014}

Institution-registered cases in 2014.

Institutions

Ageo Central General Hospital

Aichi Cancer Center

Aichi Medical University Hospital

Aizawa Hospital

Akita University Hospital

Arao Municipal Hospital

Asahi Rousai Hospital

Asahikawa Medical University Hospital

Cancer Institute Hospital of JFCR

Chiba Cancer Center

Chiba University Hospital

Chiba-ken Saiseikai Narashino Hospital

Dokkyo Medical University Hospital

Dokkyo Medical University Saitama Medical Center

Edogawa Hospital

Ehime Prefectural Central Hospital

Eijyu General Hospital

Fuchu Hospital

Fuji City General Hospital

Fujinomiya City General Hospital

Fujioka General Hospital

Fujisaki Hospital

Fujita Health University Hospital

Fukaya Red Cross Hospital

Fukui University Hospital

Fukui-ken Saiseikai Hospital

Fukuoka City Hospital

Fukuoka Shin Mizumaki Hospital

Fukuoka University Chikushi Hospital continued

Institutions

Fukuoka University Hospital

Fukushima Medical University Hospital

Fukuyama City Hospital

Fussa Hospital

Gifu Prefectural General Center

Gifu University Hospital

Gunma Prefectural Cancer Center

Gunma Saiseikai Maebashi Hospital

Gunma University Hospital

Hachinohe City Hospital

Hagi City Hospital

Hakodate City Hospital

Hakodate Goryokaku Hospital

Hakodate National Hospital

Hamamatsu University Hospital

Hannan Chuo Hospital

Hasuda Hospital

Heartlife Hospital

Higashiosaka City Medical Center

Hiraka General Hospital

Hiratsuka City Hospital

Hirosaki University Hospital

Hiroshima City Asa Hospital

Hiroshima City Hospital

Hiroshima Prefectural Hospital

Hiroshima Red Cross Hospital \& Atomic-Bomb Survivors Hospital

Hiroshima University Hospital

Hitachi General Hospital

Hofu Institute of Gastroenterology

Hokkaido University Hospital

Hospital of the University of Occupational and Environmental Health, Japan

Hyogo Cancer Center

Hyogo Prefectural Amagasaki General Medical Center

Hyogo Prefectural Nishinomiya Hospital

Ibaraki Prefectural Central Hospital

Iizuka Hospital

Ikeda City Hospital

Imari Arita Kyoritsu Hospital

International University of Health and Welfare Atami Hospital

International University of Health and Welfare Hospital

International University of Health and Welfare Mita Hospital

Isehara Kyodo Hospital

Iseikai Hospital

Ishikawa Prefectural Central Hospital

Itami City Hospital

Iwata City Hospital

Iwate Medical University Hospital 


\section{continued}

Institutions

Iwate Prefectural Central Hospital

Iwate Prefectural Chubu Hospital

JA Hiroshima General Hospital

JA Kouseiren Enshu Hospital

JA Onomichi General Hospital

Japanese Red Cross Ashikaga Hospital

Japanese Red Cross Fukuoka Hospital

Japanese Red Cross Ishinomaki Hospital

Japanese Red Cross Kitami Hospital

Japanese Red Cross Kyoto Daiichi Hospital

Japanese Red Cross Maebashi Hospital

Japanese Red Cross Medical Center

Japanese Red Cross Musashino Hospital

Japanese Red Cross Nagoya Daiichi Hospital

Japanese Red Cross Nagoya Daini Hospital

Japanese Red Cross Saitama Hospital

Japanese Red Cross Tottori Hospital

Japanese Red Cross Wakayama Medical Center

Japanese Red Cross Yamaguchi Hospital

JCHO Gunma Chuo Hospital

JCHO Kyushu Hospital

JCHO Osaka Hospital

JCHO Saitama Medical Center

Jichi Medical University Hospital

Jichi Medical University Saitama Medical Center

Juntendo University Hospital

Juntendo University Nerima Hospital

Juntendo University Shizuoka Hospital

Juntendo University Urayasu Hospital

Junwakai Memorial Hospital

Kagawa Prefectural Central Hospital

Kagawa Rosai Hospital

Kagawa University Hospital

Kagoshima City Hospital

Kagoshima University Hospital

Kakogawa Central City Hospital

Kanagawa Cancer Center

Kanagawa Prefectural Ashigarakami Hospital

Kanazawa Medical University Hospital

Kanazawa University Hospital

Kansai Denryoku Hospital

Kansai Medical University Hospital

Kansai Medical University Medical Center

Kansai Rosai Hospital

Kashiwa Kousei General Hospital

Kasugai Municipal Hospital

Kawakita General Hospital

Kawasaki Medical School Hospital

Kawasaki Medical School Kawasaki Hospital

Kawasaki Municipal Hospital continued

Institutions

Kawasaki Municipal Ida Hospital

Kawasaki Saiwai Hospital

Keio University Hospital

Keiyukai Sapporo Hospital

Kindai University Hospital

Kindai University Nara Hospital

Kinki Central Hospital

Kiryu Kousei General Hospital

Kishiwada City Hospital

Kitaakita Municipal Hospital

Kitaharima Medical Center

Kitakyushu Municipal Medical Center

Kitano Hospital

Kitasato University Hospital

Kobe City Medical Center General Hospital

Kobe University Hospital

Kochi Health Science Center

Kochi University Hospital

Kokura Memorial Hospital

Kosei Hospital

Kouseiren Takaoka Hospital

Kumagai General Hospital

Kumamoto University Hospital

Kumamoto Regional Medical Center

Kurashiki Central Hospital

Kurume University Hospital

Kyonan Medical Center Fujikawa Hospital

Kyorin University Hospital

Kyoto University Hospital

Kyoto-Katsura Hospital

Kyushu Central Hospital

Kyushu University Hospital

Machida Municipal Hospital

Matsudo City General Hospital

Matsushita Memorial Hospital

Matsuyama Red Cross Hospital

Mie University Hospital

Minamiosaka Hospital

Minoh City Hospital

Mito Red Cross Hospital

Mitsui Memorial Hospital

Miyazaki University Hospital

Moriguchi Keijinkai Hospital

Nagahama City Hospital

Nagahama Red Cross Hospital

Nagano Municipal Hospital

Nagaoka Chuo General Hospital

Nagasaki University Hospital

Nagoya City University Hospital

Nagoya City West Medical Center 
continued

Institutions

Nagoya Tokushukai General Hospital

Nagoya University Hospital

Nanpuh Hospital

Nara City Hospital

Nara Medical University Hospital

Nasu Red Cross Hospital

National Cancer Center Hospital

National Cancer Center Hospital East

National Center for Global Health and Medicine

National Defence Medical College Hospital

Nerima Hikarigaoka Hospital

New Tokyo Hospital

NHO Beppu Medical Center

NHO Chiba Medical Center

NHO Iwakuni Clinical Center

NHO Kure Medical Center

NHO Kyoto Medical Center

NHO Kyushu Cancer Center

NHO Kyushu Medical Center

NHO Matsumoto Medical Center

NHO Mito Medical Center

NHO Miyakonojo Medical Center

NHO Nagasaki Medical Center

NHO Nagoya Medical Center

NHO Okayama Medical Center

NHO Osaka Medical Center

NHO Saga Hospital

NHO Saitama Hospital

NHO Sendai Medical Center

NHO Shikoku Cancer Center

NHO Takasaki General Medical Center

NHO Tokyo Medical Center

NHO Yokohama Medical Center

Nihonkai General Hospital

Niigata Cancer Center Hospital

Niigata City General Hospital

Niigata Prefectural Shibata Hospital

Niigata University Medical \& Dental Hospital

Nikko Memorial Hospital

Nippon Medical School Chiba Hokusou Hospital

Nippon Medical School Hospital

Nippon Medical School Musashi Kosugi Hospital

Nippon Medical School Tama Nagayama Hospital

Nishi Kobe Medical Center

Northern Okinawa Medical Center

NTT Medical Center Tokyo

Numazu City Hospital

Obihiro Kousei Hospital

Ogaki Municipal Hospital

Ogikubo Hospital continued

Institutions

Ogori Daiichi General Hospital

Ohta Hospital

Ohta Nishinouchi Hospital

Oita Prefectural Hospital

Oita Red Cross Hospital

Oita University Hospital

Okayama City Hospital

Okayama Red Cross General Hospital

Okayama Saiseikai General Hospital

Okayama University Hospital

Okitama Public General Hospital

Onomichi Municipal Hospital

Osaka City General Hospital

Osaka City University Hospital

Osaka General Medical Center

Osaka International Cancer Institute

Osaka Medical College Hospital

Osaka Police Hospital

Osaka Red Cross Hospital

Osaka University Hospital

Osaki City Hospital

Otemae Hospital

Otsu City Hospital

Rinku General Medical Center

Saga Prefectural Hospital Koseikan

Saga University Hospital

Saiseikai Fukuoka General Hospital

Saiseikai Karatsu Hospital

Saiseikai Kyoto Hospital

Saiseikai Noe Hospital

Saiseikai Utsunomiya Hospital

Saiseikai Yamaguchi General Hospital

Saiseikai Yokohama Tobu Hospital

Saitama Medical University International Medical Center

Saitama Medical University Saitama Medical Center

Sakai City Medical Center

Saku Central Hospital

Sapporo Medical University Hospital

Seikei-kai Chiba Medical Center

Seirei Hamamatsu General Hospital

Sendai City Hospital

Sendai Kosei Hospital

Shiga General Hospital

Shiga University of Medical Science Hospital

Shimane University Hospital

Shin Takeo Hospital

Shinko Hospital

Shinshu University Hospital

Shizuoka Cancer Center

Shizuoka City Shizuoka Hospital 
continued

Institutions

Shizuoka General Hospital

Showa University Hospital

Southern Tohoku General Hospital

St. Luke's International Hospital

St. Marianna University School of Medicine Hospital

St. Mary's Hospital

Steel Memorial Yawata Hospital

Suita Municipal Hospital

Tachikawa Hospital

Tagawa Municipal Hospital

Takatsuki Red Cross Hospital

Teikyo University Chiba Medical Center

Teikyo University Hospital

Teikyo University Hospital Mizonokuchi

Teine Keijinkai Hospital

Tenri Hospital

The Hospital of Hyogo College of Medicine

The Jikei University Daisan Hospital

The Jikei University Hospital

Tochigi Cancer Center

Toda Central General Hospital

Toho University Ohashi Medical Center

Toho University Omori Medical Center

Toho University Sakura Medical Center

Tohoku University Hospital

Tokai University Hachioji Hospital

Tokai University Hospital

Tokai University Tokyo Hospital

Tokushima Red Cross Hospital

Tokushima University Hospital

Tokyo Dental College Ichikawa General Hospital

Tokyo Medical and Dental University Hospital

Tokyo Medical University Hachioji Medical Center

Tokyo Medical University Hospital

Tokyo Medical University Ibaraki Medical Center

Tokyo Metropolitan Cancer and Infectious Diseases Center Komagome Hospital

Tokyo Metropolitan Tama Medical Center

Tokyo University Hospital

Tokyo Women's Medical University Hospital

Tokyo Women's Medical University Medical Center East

Tokyo Women's Medical University Yachiyo Medical Center

Tonan Hospital

Toshima Hospital

Tottori Prefectural Central Hospital

Tottori University Hospital

Toyama Prefectural Central Hospital

Toyama University Hospital

Toyonaka Municipal Hospital

Toyota Kosei Hospital continued

Institutions

Toyota Memorial Hospital

Tsuchiura Kyodo Hospital

Tsukuba University Hospital

Tsuruoka Municipal Shonal Hospital

University Hospital, Kyoto Prefectural University of Medicine

University of the Ryukyus Hospital

Wakayama Medical University Hospital

Yamagata Prefectural Central Hospital

Yamagata University Hospital

Yamaguchi University Hospital

Yamanashi Prefectural Central Hospital

Yamanashi University Hospital

Yao Municipal Hospital

Yokkaichi Hospital

Yokohama City Municipal Hospital

Yokohama City University Hospital

Yokohama City University Medical Center

Yonezawa City Hospital

Yuai Memorial Hospital

(Total 344 institutions)

\section{Patient background}

Tables 1, 2, 3, 4, 5, 6, 7, 8 .

Table 1 Age and gender

\begin{tabular}{llll}
\hline Age & Male & Female & Cases $(\%)$ \\
\hline$\leq 29$ & 20 & 4 & $24(0.3)$ \\
$30-39$ & 22 & 7 & $29(0.3)$ \\
$40-49$ & 179 & 74 & $253(2.8)$ \\
$50-59$ & 995 & 230 & $1225(13.6)$ \\
$60-69$ & 2908 & 482 & $3390(37.6)$ \\
$70-79$ & 2788 & 432 & $3220(35.7)$ \\
$80-89$ & 685 & 148 & $833(9.2)$ \\
$90 \leq$ & 34 & 18 & $52(0.6)$ \\
Total & 7631 & 1395 & 9026 \\
\hline
\end{tabular}

Table 2 Performed treatment

\begin{tabular}{ll}
\hline Treatments & Cases $(\%)$ \\
\hline Surgery & $5355(59.3)$ \\
Esophagectomy & $5204(57.7)$ \\
Palliative surgery & $151(1.7)$ \\
Chemotherapy and/or radiotherapy & $4835(53.6)$ \\
Endoscopic treatment & $1529(16.9)$ \\
\hline
\end{tabular}


Table 3 Tumor location

\begin{tabular}{|c|c|c|c|c|c|}
\hline \multirow[t]{2}{*}{ Location of tumor } & \multirow{2}{*}{$\begin{array}{l}\text { Endoscopic treatment } \\
(\%)\end{array}$} & \multicolumn{2}{|l|}{ Surgery } & \multirow{2}{*}{$\begin{array}{l}\text { Chemotherapy and/or } \\
\text { radiotherapy }(\%)\end{array}$} & \multirow[t]{2}{*}{ Total $(\%)$} \\
\hline & & Esophagectomy (\%) & $\begin{array}{l}\text { Palliative sur- } \\
\text { gery }(\%)\end{array}$ & & \\
\hline Cervical & $43(2.8)$ & $185(3.6)$ & $6(4.0)$ & $305(6.3)$ & $436(4.8)$ \\
\hline Upper thoracic & $164(10.7)$ & $598(11.5)$ & $36(23.8)$ & $738(15.3)$ & $1160(12.9)$ \\
\hline Middle thoracic & $838(54.7)$ & $2386(45.8)$ & $66(43.7)$ & $2180(45.1)$ & $4200(46.5)$ \\
\hline Lower thoracic & $378(24.7)$ & $1528(29.4)$ & $35(23.2)$ & $1296(26.8)$ & $2451(27.2)$ \\
\hline EG & $68(4.4)$ & $378(7.3)$ & $7(4.6)$ & $214(4.4)$ & $531(5.9)$ \\
\hline $\mathrm{E}=\mathrm{G}$ & $24(1.6)$ & $64(1.2)$ & & $30(0.6)$ & $94(1.0)$ \\
\hline GE & $7(0.5)$ & $62(1.2)$ & & $40(0.8)$ & $85(0.9)$ \\
\hline Unknown & $7(0.5)$ & $3(0.1)$ & $1(0.7)$ & $32(0.7)$ & $69(0.8)$ \\
\hline Total & 1529 & 5204 & 151 & 4835 & 9026 \\
\hline
\end{tabular}

$E$ esophageal, $G$ gastric

Table 4 Histologic type of biopsy specimens

\begin{tabular}{|c|c|c|c|c|c|}
\hline \multirow[t]{2}{*}{ Histologic types } & \multirow{2}{*}{$\begin{array}{l}\text { Endoscopic treatment } \\
(\%)\end{array}$} & \multicolumn{2}{|l|}{ Surgery } & \multirow{2}{*}{$\begin{array}{l}\text { Chemotherapy and/or } \\
\text { radiotherapy (\%) }\end{array}$} & \multirow[t]{2}{*}{ Total $(\%)$} \\
\hline & & Esophagectomy (\%) & Palliative surgery $(\%)$ & & \\
\hline Squamous cell carcinoma & $1314(85.9)$ & $4567(87.8)$ & $143(94.7)$ & $4450(92.0)$ & $7938(87.9)$ \\
\hline Squamous cell carcinoma & $993(64.2)$ & 2484 (47.7) & 93 (61.6) & $2601(53.8)$ & $4819(53.4)$ \\
\hline Well differentiated & $104(6.8)$ & $427(8.2)$ & $12(7.9)$ & $320(6.6)$ & $640(7.1)$ \\
\hline Moderately differentiated & $172(11.2)$ & $1234(23.7)$ & $29(19.2)$ & $1098(22.7)$ & $1807(20.0)$ \\
\hline Poorly differentiated & $45(2.9)$ & $422(8.1)$ & $9(6.0)$ & $431(8.9)$ & $672(7.4)$ \\
\hline Adenocarcinoma & $41(2.7)$ & $372(7.1)$ & $3(2.0)$ & $199(4.1)$ & $492(5.5)$ \\
\hline Barrett's carcinoma & $42(2.7)$ & $96(1.8)$ & $1(0.7)$ & $25(0.5)$ & $144(1.6)$ \\
\hline Adenosquamous carcinoma & $1(0.1)$ & $10(0.2)$ & & $7(0.1)$ & $18(0.2)$ \\
\hline Mucoepidermoid carcinoma & & $2(0.0)$ & & $1(0.0)$ & $3(0.0)$ \\
\hline Basaloid carcinoma & $4(0.3)$ & $32(0.6)$ & & $19(0.4)$ & $41(0.5)$ \\
\hline Neuroendocrine tumor & & & & $1(0.0)$ & $1(0.0)$ \\
\hline Neuroendocrine carcinoma & $1(0.1)$ & $16(0.3)$ & & $34(0.7)$ & $41(0.5)$ \\
\hline Undifferentiated carcinoma & $1(0.1)$ & $4(0.1)$ & & $2(0.0)$ & $5(0.3)$ \\
\hline Malignant melanoma & & $18(0.3)$ & & $9(0.2)$ & $24(0.3)$ \\
\hline Carcinosarcoma & $1(0.1)$ & $22(0.4)$ & & $12(0.2)$ & $28(0.3)$ \\
\hline GIST & & $7(0.1)$ & & $2(0.0)$ & $8(0.1)$ \\
\hline Adenoid cystic carcinoma & & $1(0.0)$ & & & $1(0.0)$ \\
\hline Nonepithelial tumors & $2(0.1)$ & $3(0.1)$ & & $3(0.1)$ & $6(0.1)$ \\
\hline Other epithelial tumors & $36(2.4)$ & $8(0.2)$ & & $9(0.2)$ & $58(0.6)$ \\
\hline Other tumors & $26(1.7)$ & $15(0.3)$ & & $5(0.1)$ & $47(0.5)$ \\
\hline Unknown & $60(3.9)$ & $31(0.6)$ & $4(2.6)$ & $57(2.1)$ & $171(1.9)$ \\
\hline Total & 1529 & 5204 & 151 & 4835 & 9026 \\
\hline
\end{tabular}


Table 5 Depth of tumor invasion, cT (UICC TNM 7th)

\begin{tabular}{|c|c|c|c|c|c|}
\hline \multirow[t]{2}{*}{ Clinical T } & \multirow{2}{*}{$\begin{array}{l}\text { Endoscopic treatment } \\
(\%)\end{array}$} & \multicolumn{2}{|l|}{ Surgery } & \multirow{2}{*}{$\begin{array}{l}\text { Chemotherapy and/or } \\
\text { radiotherapy (\%) }\end{array}$} & \multirow[t]{2}{*}{ Total $(\%)$} \\
\hline & & Esophagectomy (\%) & $\begin{array}{l}\text { Palliative } \\
\text { surgery } \\
(\%)\end{array}$ & & \\
\hline cTX & $28(1.8)$ & $17(0.3)$ & $4(2.6)$ & $57(1.2)$ & $144(1.6)$ \\
\hline сT0 & $17(1.1)$ & $7(0.1)$ & & $3(0.1)$ & $30(0.3)$ \\
\hline cT1a & $1173(76.7)$ & $240(4.6)$ & & $112(2.3)$ & $1469(16.3)$ \\
\hline $\mathrm{cT} 1 \mathrm{~b}$ & $205(13.4)$ & $1409(27.1)$ & $2(1.3)$ & $644(13.3)$ & $1858(20.6)$ \\
\hline $\mathrm{cT} 2$ & $9(0.6)$ & $867(16.7)$ & $5(3.3)$ & $667(13.8)$ & $1086(12.0)$ \\
\hline cT3 & $46(3.0)$ & $2310(44.4)$ & $62(41.1)$ & $2367(49.0)$ & $3250(36.0)$ \\
\hline cT4a & $10(0.7)$ & $164(3.2)$ & $13(8.6)$ & $317(6.6)$ & $404(4.5)$ \\
\hline $\mathrm{cT} 4 \mathrm{~b}$ & $41(2.7)$ & $190(3.7)$ & $65(43.0)$ & $668(13.8)$ & $785(8.7)$ \\
\hline Total & 1529 & 5204 & 151 & 4835 & 9026 \\
\hline
\end{tabular}

Table 6 Lymph-node metastasis, cN (UICC TNM 7th)

\begin{tabular}{|c|c|c|c|c|c|}
\hline \multirow[t]{2}{*}{ Clinical N } & \multirow{2}{*}{$\begin{array}{l}\text { Endoscopic treatment } \\
(\%)\end{array}$} & \multicolumn{2}{|l|}{ Surgery } & \multirow{2}{*}{$\begin{array}{l}\text { Chemotherapy and/or } \\
\text { radiotherapy (\%) }\end{array}$} & \multirow[t]{2}{*}{ Total $(\%)$} \\
\hline & & Esophagectomy (\%) & $\begin{array}{l}\text { Palliative } \\
\text { surgery } \\
(\%)\end{array}$ & & \\
\hline $\mathrm{cNO}$ & $1426(93.3)$ & $2390(45.9)$ & $20(13.2)$ & $1310(27.1)$ & $4399(48.7)$ \\
\hline $\mathrm{cN} 1$ & $50(3.3)$ & $1825(35.1)$ & $60(39.7)$ & 1914 (39.6) & $2627(29.1)$ \\
\hline $\mathrm{cN} 2$ & $33(2.2)$ & 867 (16.7) & $56(37.1)$ & $1257(26.0)$ & $1567(17.4)$ \\
\hline cN3 & $20(1.3)$ & $122(2.3)$ & $15(9.9)$ & $354(7.3)$ & $433(4.8)$ \\
\hline Total & 1529 & 5204 & 151 & 4835 & 9026 \\
\hline
\end{tabular}

Table 7 Distant metastasis, cM (UICC TNM 7th)

\begin{tabular}{|c|c|c|c|c|c|}
\hline \multirow[t]{2}{*}{ Clinical M } & \multirow{2}{*}{$\begin{array}{l}\text { Endoscopic treatment } \\
(\%)\end{array}$} & \multicolumn{2}{|l|}{ Surgery } & \multirow{2}{*}{$\begin{array}{l}\text { Chemotherapy and/or } \\
\text { radiotherapy (\%) }\end{array}$} & \multirow[t]{2}{*}{ Total (\%) } \\
\hline & & Esophagectomy (\%) & Palliative surgery $(\%)$ & & \\
\hline cM0 & 1494 (97.7) & $5036(96.8)$ & $108(71.5)$ & $4210(85.2)$ & $8148(90.3)$ \\
\hline cM1 & $35(2.3)$ & $168(3.2)$ & $43(28.5)$ & $715(14.8)$ & $878(9.7)$ \\
\hline Total & 1529 & 5204 & 151 & 4835 & 9026 \\
\hline
\end{tabular}

Table 8 Clinical Stage (UICC TNM 7th)

\begin{tabular}{|c|c|c|c|c|c|}
\hline \multirow[t]{2}{*}{ Clinical stage } & \multirow{2}{*}{$\begin{array}{l}\text { Endoscopic treatment } \\
(\%)\end{array}$} & \multicolumn{2}{|l|}{ Surgery } & \multirow{2}{*}{$\begin{array}{l}\text { Chemotherapy and/or } \\
\text { radiotherapy (\%) }\end{array}$} & \multirow[t]{2}{*}{ Total (\%) } \\
\hline & & Esophagectomy (\%) & $\begin{array}{l}\text { Palliative surgery } \\
(\%)\end{array}$ & & \\
\hline Stage IA & $1363(89.1)$ & $1307(25.1)$ & $2(1.3)$ & $471(9.7)$ & $2899(32.1)$ \\
\hline Stage IB & $5(0.3)$ & $458(8.8)$ & $2(1.3)$ & $282(5.8)$ & $558(6.2)$ \\
\hline Stage IIA & $10(0.7)$ & $531(10.2)$ & $6(4.0)$ & $400(8.3)$ & $649(7.2)$ \\
\hline Stage IIB & $15(1.0)$ & $577(11.1)$ & $1(0.7)$ & $449(9.3)$ & $680(7.5)$ \\
\hline Stage IIIA & $14(0.9)$ & $1195(23.0)$ & $21(13.9)$ & $1078(22.3)$ & $1499(16.6)$ \\
\hline Stage IIIB & $8(0.5)$ & $560(10.8)$ & $16(10.6)$ & $567(11.7)$ & $733(8.1)$ \\
\hline Stage IIIC & $35(2.3)$ & $385(7.4)$ & $57(37.7)$ & 839 (17.4) & $997(11.0)$ \\
\hline Stage IV & $35(2.3)$ & $168(3.2)$ & $43(28.5)$ & $715(14.8)$ & $878(9.7)$ \\
\hline Unknown & $44(2.9)$ & $23(0.4)$ & $3(2.0)$ & $34(0.7)$ & $133(1.5)$ \\
\hline Total & 1529 & 5204 & 151 & 4835 & 9026 \\
\hline
\end{tabular}




\section{Results of endoscopically treated patients} in 2014

Tables 9, 10, 11, and Figs. 1, 2, 3.

Table 9 Details of endoscopic treatment for curative intent

Fig. 1 Survival of patients treated with EMR/ESD
Table 10 Complications of EMR/ESD

\begin{tabular}{ll}
\hline Treatment details & Cases (\%) \\
\hline EMR & $104(7.1)$ \\
EMR + YAG laser & $1(0.1)$ \\
EMR + MCT/RFA & \\
ESD & $1265(86.0)$ \\
ESD+EMR & $80(5.4)$ \\
ESD + PDT & \\
ESD+YAG laser & $2(0.1)$ \\
PDT & $3(0.2)$ \\
YAG laser & $16(1.1)$ \\
Total & 1471 \\
\hline
\end{tabular}

EMR endoscopic mucosal resection, $P D T$ photodynamic therapy, $Y A G$ yttrium aluminum garnet, $M C T$ microwave coagulation therapy, $E S D$ endoscopic submucosal dissection mens
Complications of $\quad$ Cases $(\%)$ EMR/ESD

\begin{tabular}{ll}
\hline None & $1384(95.8)$ \\
Perforation & $12(0.8)$ \\
Bleeding & $3(0.2)$ \\
Mediastinitis & $5(0.3)$ \\
Stenosis & $41(2.8)$ \\
Others & \\
Unknown & \\
Total & 1445 \\
\hline
\end{tabular}

Table 11 Pathologic depth of tumor invasion of MER/ESD speci-

\begin{tabular}{ll}
\hline Pathological depth of tumor invasion $(\mathrm{pT})$ & Cases $(\%)$ \\
\hline pTX & $17(1.2)$ \\
pT0 & $68(0.5)$ \\
pT1a & $1127(82.8)$ \\
pT1b & $238(15.0)$ \\
pT2 & \\
pT3 & $2(0.1)$ \\
Total & 1452 \\
\hline
\end{tabular}

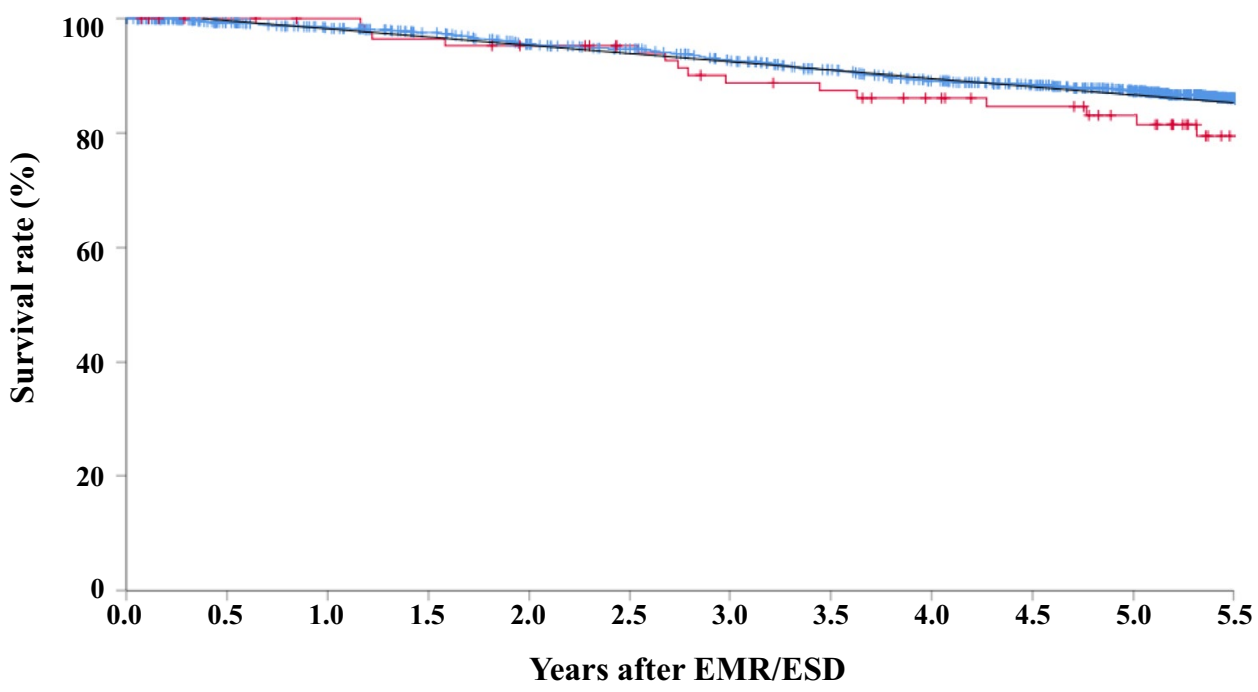

\begin{tabular}{lccccc}
\hline & \multicolumn{5}{c}{ Years after EMR/ESD } \\
\cline { 2 - 6 } & 1 & 2 & 3 & 4 & 5 \\
\hline Total & $98.5 \%$ & $95.5 \%$ & $92.4 \%$ & $89.0 \%$ & $87.1 \%$ \\
Complete resection & $98.4 \%$ & $95.6 \%$ & $92.6 \%$ & $89.2 \%$ & $87.3 \%$ \\
Incomplete resection & $\mathbf{1 0 0 . 0 \%}$ & $\mathbf{9 5 . 2} \%$ & $\mathbf{8 8 . 9} \%$ & $\mathbf{8 6 . 2} \%$ & $\mathbf{8 3 . 1} \%$ \\
\hline
\end{tabular}


Fig. 2 Survival of patients treated with EM/ESD according to the pathological depth of tumor invasion, pT (JES 10th)

Fig. 3 Survival of patients treated with EMR/ESD according to the lymphatic and venous invasion

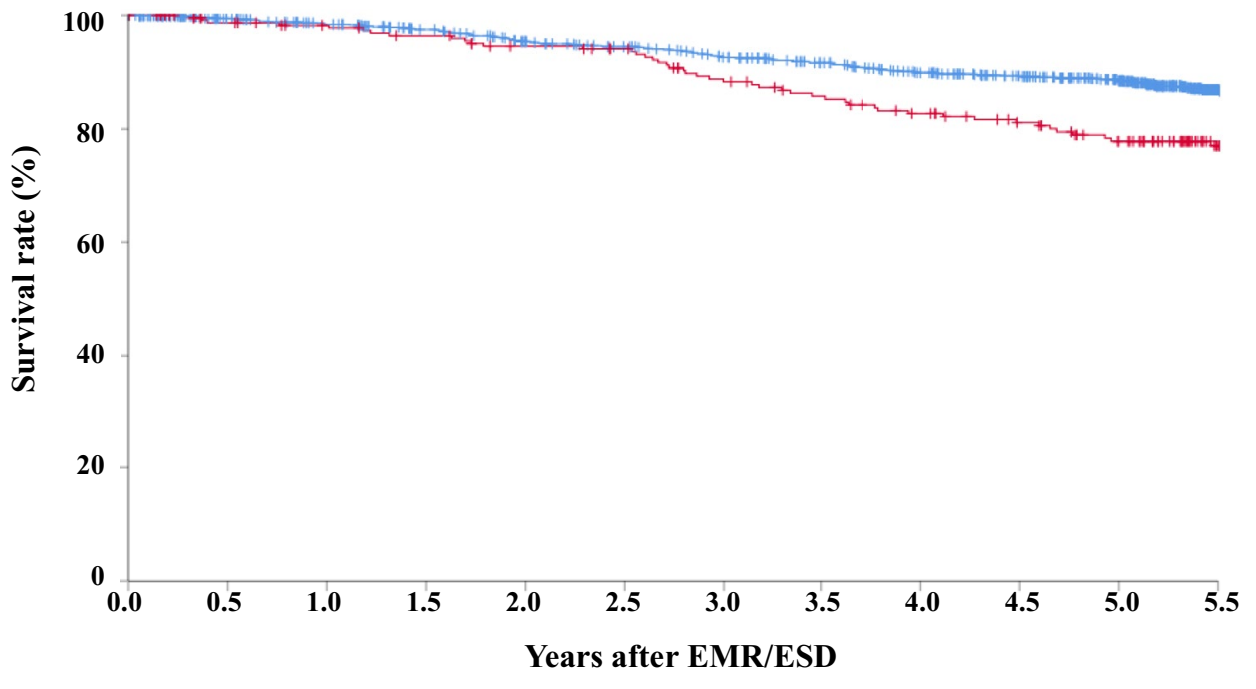

pT1a $(n=1114) \quad$ pT1b $(n=237)$

\begin{tabular}{lccccc}
\hline & \multicolumn{5}{c}{ Years after EMR/ESD } \\
\cline { 2 - 6 } & 1 & 2 & 3 & 4 & 5 \\
\hline pT1a & $98.4 \%$ & $95.4 \%$ & $92.6 \%$ & $89.9 \%$ & $88.5 \%$ \\
pT1b & $98.2 \%$ & $94.6 \%$ & $\mathbf{8 8 . 8} \%$ & $\mathbf{8 2 . 8} \%$ & $\mathbf{7 7 . 9 \%}$ \\
\hline
\end{tabular}

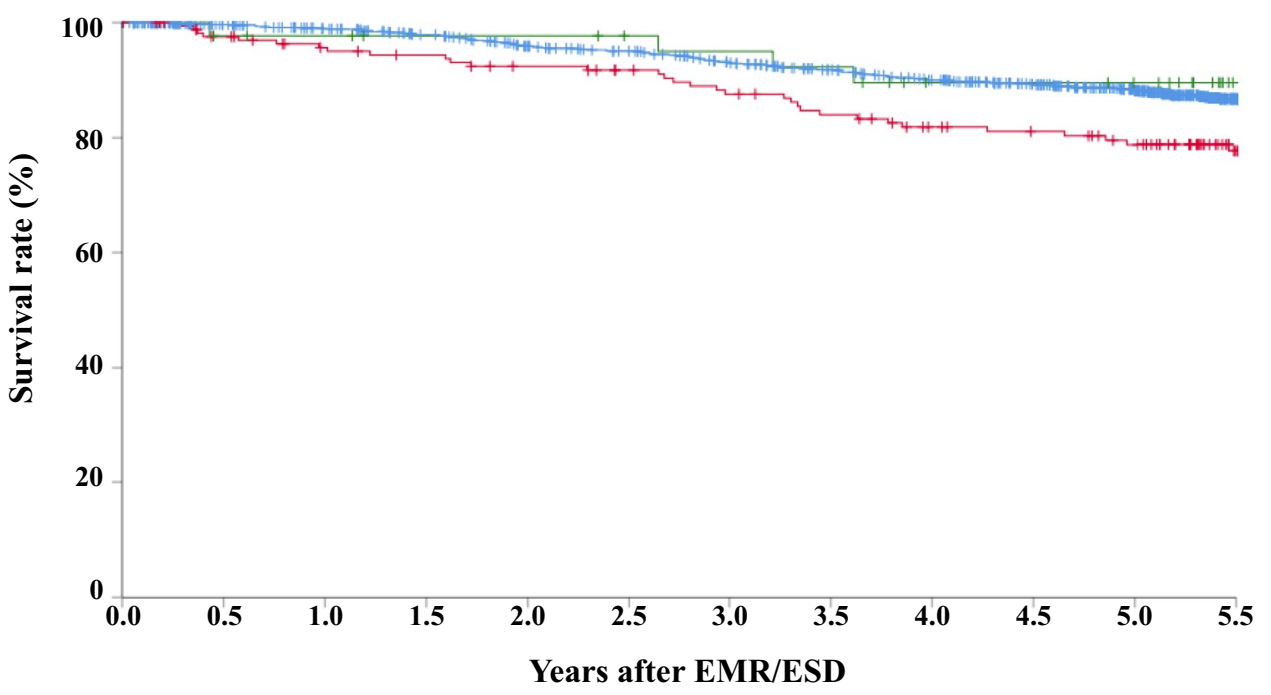

ly- and $v-(n=1227) \quad l y+$ or $v+(n=168)$

Unknown $(n=43)$

\begin{tabular}{lccccc}
\hline & \multicolumn{5}{c}{ Years after EMR/ESD } \\
\cline { 2 - 6 } & 1 & 2 & 3 & 4 & 5 \\
\hline ly0_and_v0 & $98.9 \%$ & $95.9 \%$ & $93.0 \%$ & $90.0 \%$ & $88.2 \%$ \\
ly1-3_or_v1-3 & $95.7 \%$ & $92.4 \%$ & $\mathbf{8 7 . 6} \%$ & $\mathbf{8 1 . 9} \%$ & $\mathbf{7 8 . 8} \%$ \\
Unknown & $97.6 \%$ & $97.6 \%$ & $95.0 \%$ & $\mathbf{8 9 . 2} \%$ & $\mathbf{8 9 . 2} \%$ \\
\hline
\end{tabular}




\section{Results in patients treated with chemotherapy and/or radiotherapy in 2014}

Tables 12, 13 and Figs. 4, 5, 6.

Table 12 Dose of irradiation (non-surgically treated cases)

\begin{tabular}{|c|c|c|c|c|c|c|}
\hline \multirow{2}{*}{$\begin{array}{l}\text { Dose of irradiation } \\
\text { (Gy) }\end{array}$} & \multicolumn{2}{|l|}{ Definitive } & \multirow[t]{2}{*}{ Palliative (\%) } & \multirow[t]{2}{*}{ Recurrence (\%) } & \multirow[t]{2}{*}{ Others (\%) } & \multirow[t]{2}{*}{ Total $(\%)$} \\
\hline & Radiation alone (\%) & With chemotherapy (\%) & & & & \\
\hline-29 & $2(1.2)$ & $16(1.7)$ & $26(8.4)$ & $2(6.3)$ & $3(37.5)$ & $49(3.3)$ \\
\hline $30-39$ & $3(1.8)$ & $17(1.8)$ & $53(17.1)$ & $5(15.6)$ & & $78(5.6)$ \\
\hline $40-49$ & $5(3.0)$ & $34(3.5)$ & $56(18.1)$ & $4(12.5)$ & $2(25.0)$ & $101(6.8)$ \\
\hline $50-59$ & $26(15.8)$ & $246(25.5)$ & $77(24.8)$ & $8(25.0)$ & $1(12.5)$ & $359(24.2)$ \\
\hline $60-69$ & $124(75.2)$ & $620(64.4)$ & $90(29.0)$ & $11(34.4)$ & $2(25.0)$ & $849(57.3)$ \\
\hline $70-$ & $4(2.4)$ & $28(2.9)$ & $5(1.6)$ & $2(.3)$ & & $39(2.6)$ \\
\hline Unknown & $1(0.6)$ & $2(0.2)$ & $3(1.0)$ & & & $6(0.4)$ \\
\hline Total & 165 & 963 & 310 & 32 & 8 & 1481 \\
\hline Median (min-max) & $60.0(10.0-70.0)$ & $60.0(2.0-92.0)$ & $50.0(2.0-90.0)$ & $50.4(8.0-70.0)$ & $60.0(50.0-63.4)$ & $60.0(2.0-92.0)$ \\
\hline
\end{tabular}

Table 13 Dose of irradiation (surgically treated cases)

\begin{tabular}{lll}
\hline Dose of & Preoperative & Postoperative \\
\hline irradiation (Gy) & irradiation $(\%)$ & irradiation (\%) \\
-29 & $12(3.7)$ & \\
$30-39$ & $55(16.9)$ & $3(5.0)$ \\
$40-49$ & $199(61.0)$ & $9(15.0)$ \\
$50-59$ & $40(12.3)$ & $20(33.3)$ \\
$60-69$ & $16(4.9)$ & $24(40.0)$ \\
$70-$ & $1(0.3)$ & $3(5.0)$ \\
Unknown & $3(0.9)$ & $1(1.7)$ \\
Total & 326 & 60 \\
Median (min-max) & $40.0(1.8-70.0)$ & $54.0(30.0-97.5)$
\end{tabular}


Fig. 4 Survival of patients treated with chemotherapy and/ or radiotherapy

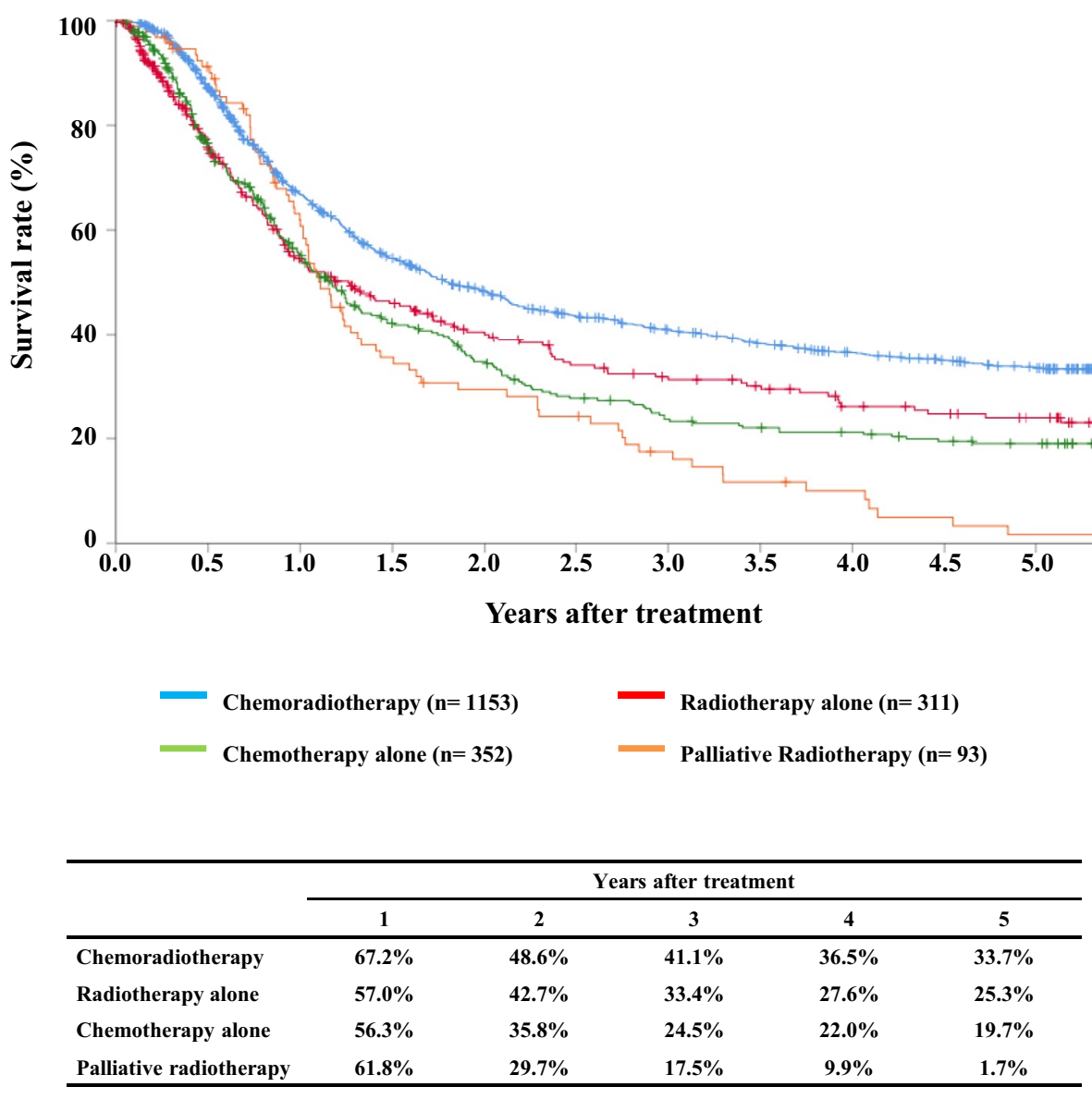


Fig. 5 Survival of patients treated with definitive chemoradiotherapy according to the clinical stage (UICC TNM 7th)
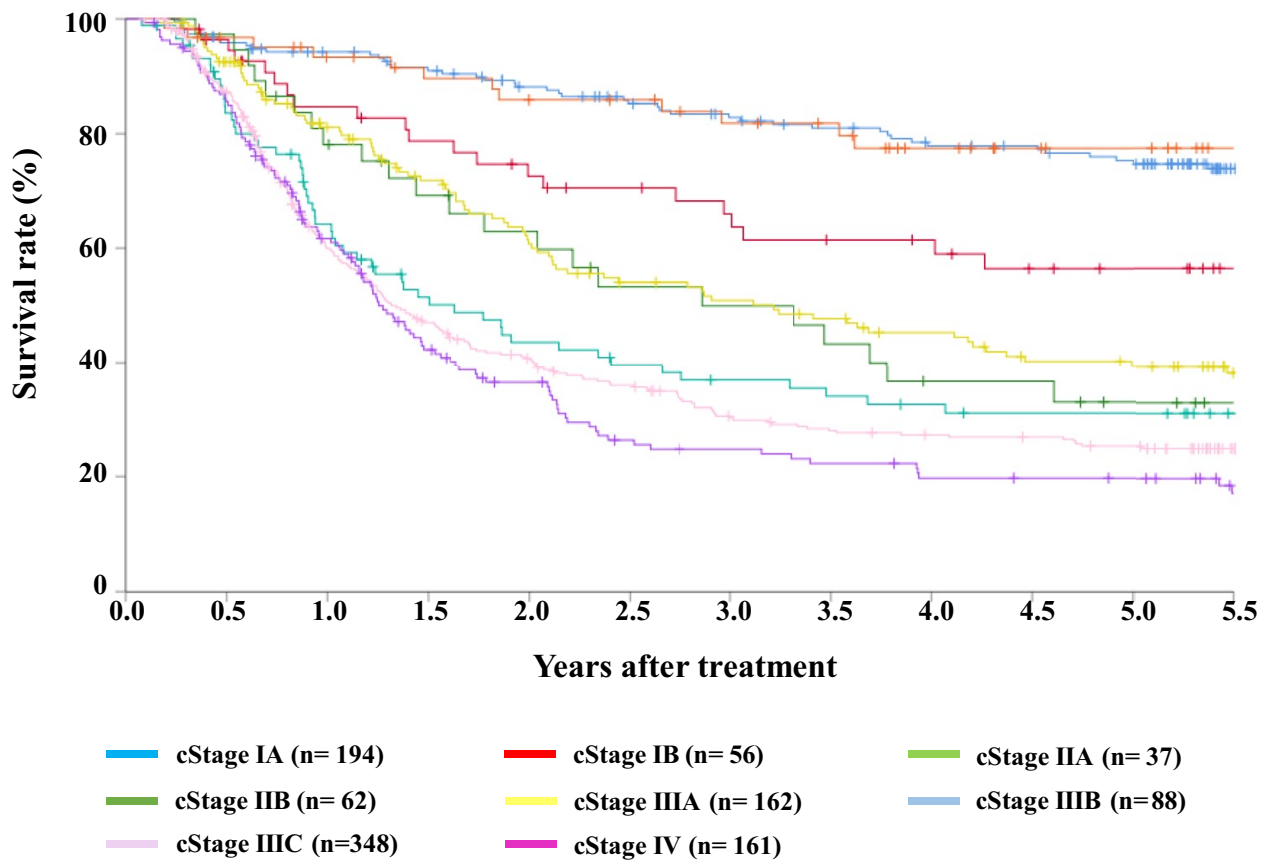

\begin{tabular}{lccccc}
\hline & \multicolumn{5}{c}{ Years after treatment } \\
\cline { 2 - 6 } & 1 & 2 & 3 & 4 & 5 \\
\hline cStage IA & $94.2 \%$ & $88.0 \%$ & $82.8 \%$ & $77.8 \%$ & $75.3 \%$ \\
cStage IB & $85.0 \%$ & $72.9 \%$ & $66.4 \%$ & $61.6 \%$ & $56.3 \%$ \\
cStage IIA & $78.1 \%$ & $63.3 \%$ & $50.4 \%$ & $36.5 \%$ & $32.4 \%$ \\
cStage IIB & $93.2 \%$ & $85.9 \%$ & $82.0 \%$ & $77.5 \%$ & $77.5 \%$ \\
cStage IIIA & $81.1 \%$ & $61.0 \%$ & $51.1 \%$ & $45.4 \%$ & $39.4 \%$ \\
cStage IIIB & $64.7 \%$ & $44.2 \%$ & $37.3 \%$ & $32.9 \%$ & $31.4 \%$ \\
cStage IIIC & $60.8 \%$ & $41.2 \%$ & $30.6 \%$ & $27.6 \%$ & $25.6 \%$ \\
cStage IV & $61.5 \%$ & $36.2 \%$ & $24.5 \%$ & $19.5 \%$ & $19.5 \%$ \\
\hline
\end{tabular}


Fig. 6 Survival of patients who underwent radiotherapy alone according to the clinical stage (UICC TNM 7th)

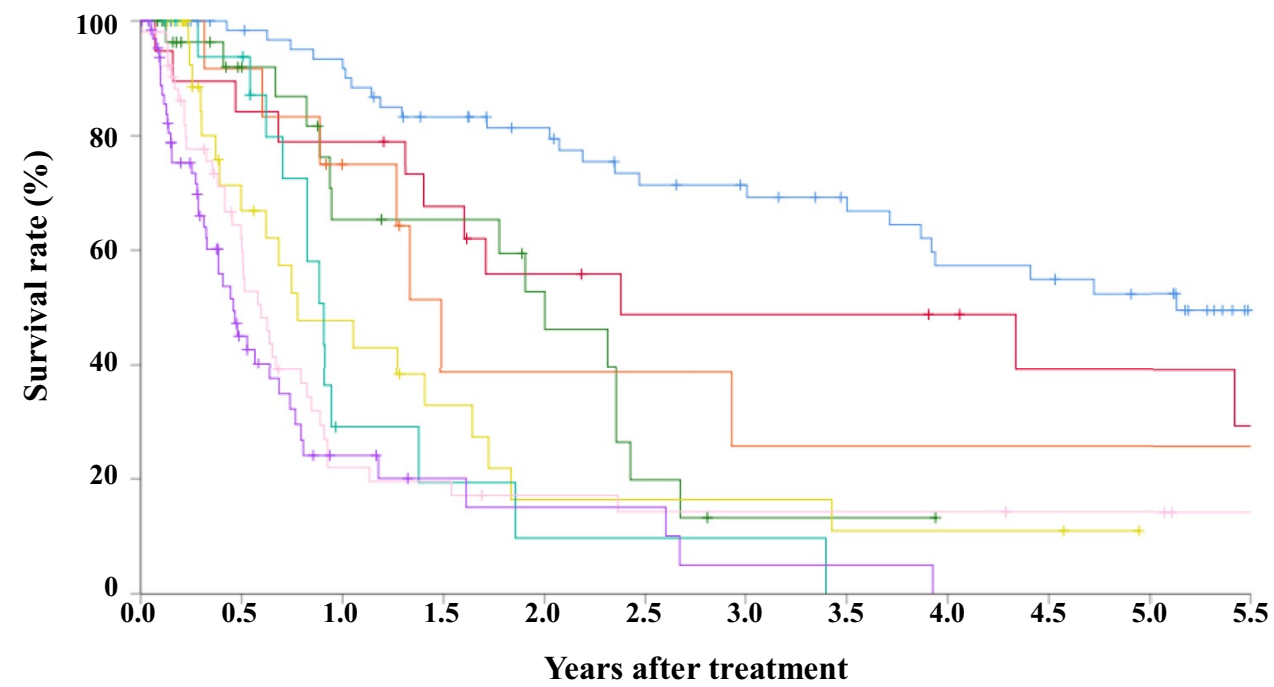

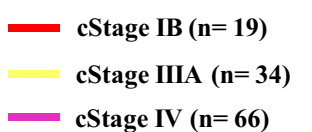

cStage IV $(n=66)$

\begin{tabular}{lccccc}
\hline & \multicolumn{5}{c}{ Years after treatment } \\
\cline { 2 - 6 } & 1 & 2 & 3 & 4 & 5 \\
\hline cStage IA & $92.1 \%$ & $81.4 \%$ & $71.2 \%$ & $57.7 \%$ & $52.6 \%$ \\
cStage IB & $78.9 \%$ & $56.4 \%$ & $49.8 \%$ & $49.8 \%$ & $40.7 \%$ \\
cStage IIA & $71.4 \%$ & $58.4 \%$ & $11.7 \%$ & $11.7 \%$ & - \\
cStage IIB & $72.7 \%$ & $39.2 \%$ & $26.1 \%$ & $26.1 \%$ & $26.1 \%$ \\
cStage IIIA & $57.1 \%$ & $21.1 \%$ & $21.1 \%$ & $14.0 \%$ & $14.0 \%$ \\
cStage IIIB & $35.5 \%$ & $11.8 \%$ & $11.8 \%$ & $0.0 \%$ & - \\
cStage IIIC & $27.1 \%$ & $20.7 \%$ & $17.3 \%$ & $17.3 \%$ & $17.3 \%$ \\
cStage IV & $31.5 \%$ & $21.0 \%$ & $7.0 \%$ & $0.0 \%$ & - \\
\hline
\end{tabular}

\section{Results in patients who underwent esophagectomy in 2014}

Tables 14, 15, 16, 17, 18, 19, 20, 21, 22, 23, 24, 25, 26, 27 , and Figs. 7, 8, 9, 10, 11, 12, 13, 14, 15
Table 14 Treatment modalities of esophagectomy

\begin{tabular}{ll}
\hline Treatment modalities & Cases $(\%)$ \\
\hline Esophagectomy alone & $2307(44.3)$ \\
Esophagectomy + postoperative chemotherapy & $387(7.4)$ \\
Esophagectomy + postoperative chemoradiotherapy & $109(2.1)$ \\
Esophagectomy + postoperative radiotherapy & $39(0.7)$ \\
Preoperative chemotherapy + Esophagectomy & $1784(34.3)$ \\
Preoperative chemoradiotherapy + Esophagectomy & $335(6.4)$ \\
Definitive radiotherapy + Esophagectomy & $6(0.1)$ \\
Definitive chemoradiotherapy + Esophagectomy & $124(2.4)$ \\
Others & $113(2.2)$ \\
Total & 5204 \\
\hline
\end{tabular}


Table 15 Tumor location

\begin{tabular}{ll}
\hline Locations & Cases $(\%)$ \\
\hline Cervical & $209(3.8)$ \\
Upper thoracic & $655(12.0)$ \\
Middle thoracic & $2448(44.9)$ \\
Lower thoracic & $1570(28.8)$ \\
EG & $380(7.0)$ \\
E =G & $98(1.8)$ \\
GE & $80(1.5)$ \\
Unknown & $11(0.2)$ \\
Total & 5451 \\
\hline
\end{tabular}

Table 16 Approaches to tumor resection

\begin{tabular}{ll}
\hline Approaches & Cases $(\%)$ \\
\hline Cervical & $176(3.4)$ \\
Right thoracic & $4492(86.3)$ \\
Left thoracic & $54(1.0)$ \\
Left thoracoabdominal & $82(1.6)$ \\
Abdominal & $187(3.6)$ \\
Transhiatal lower esophagectomy & $133(2.6)$ \\
Transhiatal thoracic esophagectomy & $64(1.2)$ \\
Sternotomy & $6(0.1)$ \\
Others & $7(0.1)$ \\
Unknown & $3(0.1)$ \\
Total & 5204 \\
\hline
\end{tabular}

Thoracic includes thoracotomy and thoracoscopic. Abdominal includes laparotomy and laparoscopic
Table 17 Video-assisted surgery

\begin{tabular}{ll}
\hline Video-assisted surgery & Cases $(\%)$ \\
\hline None & $2330(44.6)$ \\
Thoracoscopy & $1206(23.2)$ \\
Thoracoscopy + laparoscopy & $1281(24.6)$ \\
Thoracoscopy + laparoscopy + mediastinoscopy & $9(0.2)$ \\
Thoracoscopy + laparoscopy + other & \\
Thoracoscopy + mediastinoscopy & $1(0.0)$ \\
Thoracoscopy + other & $4(0.1)$ \\
Laparoscopy & $265(5.1)$ \\
Laparoscopy + mediastinoscopy & $41(0.8)$ \\
Laparoscopy + mediastinoscopy +other & $1(0.0)$ \\
Mediastinoscopy & $49(0.9)$ \\
Laparoscopy + other & $1(0.0)$ \\
Others & $15(0.3)$ \\
Unknown & $1(0.0)$ \\
Total & 5204 \\
\hline
\end{tabular}

Table 18 Fields of lymph-node dissection according to the location of tumor

\begin{tabular}{|c|c|c|c|c|c|c|c|c|c|}
\hline $\begin{array}{l}\text { Field of lymphad- } \\
\text { enectomy }\end{array}$ & Cervical & Upper thoracic & Middle thoracic & Lower thoracic & Abdominal & $\mathrm{E}=\mathrm{G}$ & GE & Unknown & Total \\
\hline None & $8(4.2)$ & $15(2.5)$ & $46(1.9)$ & $26(1.7)$ & $4(1.1)$ & $1(1.1)$ & $4(6.0)$ & & $104(2.0)$ \\
\hline $\mathrm{C}$ & $47(24.5)$ & $11(1.8)$ & $33(1.4)$ & $14(0.9)$ & & & & & $105(2.0)$ \\
\hline $\mathrm{C}+\mathrm{UM}$ & $21(10.9)$ & $1(0.2)$ & $2(0.1)$ & & $1(0.3)$ & & & & $25(0.5)$ \\
\hline $\mathrm{C}+\mathrm{UM}+\mathrm{MLM}$ & $4(2.1)$ & $21(3.4)$ & $50(2.1)$ & $12(0.8)$ & $1(0.3)$ & & & & $88(1.7)$ \\
\hline $\mathrm{C}+\mathrm{UM}+\mathrm{MLM}+\mathrm{A}$ & $83(43.2)$ & 394 (64.6) & 1205 (64.6) & 577 (37.7) & $43(12.0)$ & $6(6.8)$ & $6(9.0)$ & $1(50.0)$ & $2315(44.5)$ \\
\hline $\mathrm{C}+\mathrm{UM}+\mathrm{A}$ & $6(3.1)$ & $10(1.6)$ & $22(0.9)$ & $10(0.7)$ & $1(0.3)$ & & & & $49(0.9)$ \\
\hline $\mathrm{C}+\mathrm{MLM}$ & $1(0.5)$ & $1(0.2)$ & & & & & & & $2(0.0)$ \\
\hline $\mathrm{C}+\mathrm{MLM}+\mathrm{A}$ & $1(0.5)$ & $3(0.5)$ & $15(0.4)$ & $6(0.4)$ & $3(0.8)$ & $1(1.1)$ & & & $29(0.6)$ \\
\hline $\mathrm{C}+\mathrm{A}$ & $4(2.1)$ & $1(0.2)$ & $1(0.0)$ & $2(0.1)$ & & & & & $8(0.2)$ \\
\hline UM & $2(1.0)$ & $4(0.7)$ & $11(0.5)$ & $2(0.1)$ & & & & & $19(0.4)$ \\
\hline $\mathrm{UM}+\mathrm{MLM}$ & $3(1.6)$ & $8(1.3)$ & $40(1.7)$ & $27(1.8)$ & $4(1.1)$ & & & & $82(1.6)$ \\
\hline $\mathrm{UM}+\mathrm{MLM}+\mathrm{A}$ & $5(2.6)$ & $125(20.5)$ & 847 (35.9) & $675(44.1)$ & $115(32.2)$ & $24(27.3)$ & $3(4.5)$ & $1(50.0)$ & $1795(34.5)$ \\
\hline $\mathrm{UM}+\mathrm{A}$ & & $5(0.8)$ & $14(0.6)$ & $9(0.6)$ & $3(0.8)$ & $1(1.1)$ & & & $32(0.6)$ \\
\hline MLM & & $2(0.3)$ & $11(0.5)$ & $15(1.0)$ & $3(0.8)$ & $2(2.3)$ & $1(1.5)$ & & $34(0.7)$ \\
\hline $\mathrm{MLM}+\mathrm{A}$ & & $4(0.7)$ & $48(2.0)$ & $130(8.5)$ & 139 (38.9) & $39(44.3)$ & $33(49.3)$ & & $399(7.7)$ \\
\hline A & $1(0.5)$ & $5(0.8)$ & $14(0.6)$ & 24 (1.6) & $40(11.2)$ & $14(15.9)$ & $20(29.9)$ & & $118(2.3)$ \\
\hline Total & 192 & 610 & 2359 & 1529 & 357 & 88 & 67 & 2 & 5204 \\
\hline
\end{tabular}

$C$ bilateral cervical nodes, $U M$ upper mediastinal nodes, $M L M$ middle-lower mediastinal nodes, $A$ abdominal nodes 
Table 19 Reconstruction route

\begin{tabular}{ll}
\hline Route & Cases $(\%)$ \\
\hline None & $47(0.9)$ \\
Subcutaneous & $345(6.6)$ \\
Retrosternal & $2315(44.5)$ \\
Posterior mediastinal & $1920(36.9)$ \\
Intrathoracic & $465(8.9)$ \\
Cervical & $65(1.2)$ \\
Others & $41(0.8)$ \\
Unknown & $6(0.1)$ \\
Total & 5204 \\
\hline
\end{tabular}

Table 20 Organs used for reconstruction

Table 21 Histological classification

\begin{tabular}{ll}
\hline Histological classification & Cases $(\%)$ \\
\hline Squamous cell carcinoma & $4324(83.1)$ \\
Squamous cell carcinoma & $751(14.4)$ \\
Well differentiated & $764(14.7)$ \\
Moderately differentiated & $2172(41.7)$ \\
Poorly differentiated & $637(12.2)$ \\
Adenocarcinoma & $347(6.7)$ \\
Barrett's carcinoma & $113(2.2)$ \\
Adenosquamous carcinoma & $29(0.6)$ \\
Mucoepidermoid carcinoma & $6(0.1)$ \\
Basaloid carcinoma & $82(1.6)$ \\
Neuroendocrine tumor & $2(0.0)$ \\
Neuroendocrine carcinoma & $25(0.5)$ \\
Undifferentiated carcinoma & $5(0.1)$ \\
Malignant melanoma & $19(0.4)$ \\
Carcinosarcoma & $37(0.7)$ \\
GIST & $7(0.1)$ \\
Adenoid cystic carcinoma & $1(0.0)$ \\
Sarcoma & $2(0.0)$ \\
Other carcinomas & $8(0.2)$ \\
Other tumors & $54(1.0)$ \\
Unknown & $143(2.7)$ \\
Total & 5204 \\
\hline
\end{tabular}

Table 22 Pathological depth of tumor invasion, pT (JES 10th)

\begin{tabular}{ll}
\hline Organs & Cases $(\%)$ \\
\hline None & $85(1.6)$ \\
Whole stomach & $105(2.0)$ \\
Gastric tube & $4425(84.3)$ \\
Jejunum & $272(5.2)$ \\
Free jejunum & $119(2.3)$ \\
Colon & $197(3.8)$ \\
Free colon & $10(0.2)$ \\
Others & $36(0.7)$ \\
Total organs & 5249 \\
Total cases & 5119 \\
\hline
\end{tabular}

Table 24 Pathological grading of lymph-node metastasis, pN

(UICC TNM 7th)

Table 23 Pathological grading of lymph-node metastasis, $\mathrm{pN}$ (JES 10th)

Table 25 Pathological findings of distant organ metastasis, $\mathrm{pM}$ (JES 10th)

Table 26 Residual tumor
Pathological depth Cases (\%) of tumor invasion

\begin{tabular}{ll}
\hline pTx & $42(0.8)$ \\
pT0 & $227(4.4)$ \\
pT1a & $645(12.4)$ \\
pT1b & $1475(28.3)$ \\
pT2 & $590(11.3)$ \\
pT3 & $1962(37.7)$ \\
pT4a & $141(2.7)$ \\
pT4b & $122(2.3)$ \\
Total & 5204 \\
\hline
\end{tabular}

\begin{tabular}{ll}
\hline $\begin{array}{l}\text { Lymph-node } \\
\text { metastasis }\end{array}$ & Cases $(\%)$ \\
\hline pN0 & $2568(49.3)$ \\
pN1 & $962(18.5)$ \\
pN2 & $966(18.6)$ \\
pN3 & $371(7.1)$ \\
pN4 & $321(6.2)$ \\
Unknown & $16(0.3)$ \\
Total & 5204 \\
\hline
\end{tabular}

\begin{tabular}{ll}
\hline $\begin{array}{l}\text { Lymph-node } \\
\text { metastasis }\end{array}$ & Cases (\%) \\
\hline pN0 & $2611(50.2)$ \\
pN1 (1-2) & $1397(26.8)$ \\
pN2 (3-6) & $787(15.1)$ \\
pN3 (7-) & $373(7.2)$ \\
Unknown & $36(0.7)$ \\
Total & 5204 \\
\hline
\end{tabular}

Distant metastasis Cases (\%) (M)

\begin{tabular}{ll}
\hline MX & $110(2.1)$ \\
M0 & $4998(96.0)$ \\
M1 & $96(1.8)$ \\
Total & 5204 \\
\hline
\end{tabular}

\begin{tabular}{ll}
\hline $\begin{array}{l}\text { Residual tumor } \\
(\mathrm{R})\end{array}$ & Cases (\%) \\
\hline RX & $95(1.8)$ \\
R0 & $4663(89.6)$ \\
R1 & $257(4.9)$ \\
R2 & $189(3.6)$ \\
Total & 5204 \\
\hline
\end{tabular}


Table 27 Cause of death

\begin{tabular}{ll}
\hline Cause of death & Cases (\%) \\
\hline Death due to recurrence & $1806(62.0)$ \\
Death due to other cancer & $231(7.9)$ \\
Death due to other disease (with recurrence) & $65(2.2)$ \\
Death due to other disease (without recurrence) & $402(13.8)$ \\
Death due to other disease (recurrence unknown) & $12(0.4)$ \\
Operative death* & $39(1.3)$ \\
Postoperative hospital death** & $65(2.2)$ \\
Unknown & $291(10.0)$ \\
Total of death cases & 2911 \\
\hline
\end{tabular}

Operative mortality rate: $0.75 \%$

*Operative death means death within 30 days after operation in or out of hospital

**Hospital death is defined as death during the same hospitalization, regardless of department at time of death. Hospital mortality rate: $2.0 \%$

Follow-up period (months)

\begin{tabular}{ll}
\hline Median (min.-max.) & $55.29(0.07-78.78)$
\end{tabular}

Fig. 7 Survival of patients who underwent esophagectomy

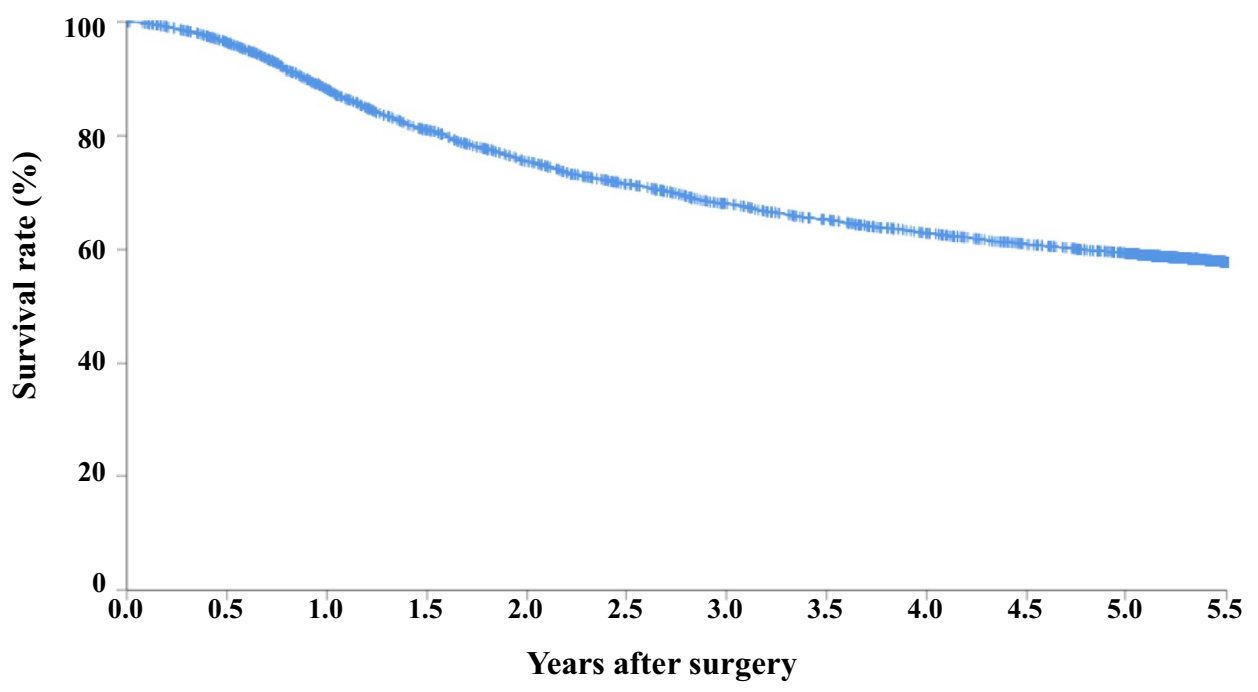

Esophagectomy $(\mathbf{n}=\mathbf{5 1 0 9})$

\begin{tabular}{lccccc}
\hline & \multicolumn{5}{c}{ Years after surgery } \\
\cline { 2 - 6 } & 1 & 2 & 3 & 4 & 5 \\
\hline Esophagectomy & $88.2 \%$ & $75.5 \%$ & $68.1 \%$ & $62.9 \%$ & $59.3 \%$ \\
\hline
\end{tabular}


Fig. 8 Survival of patients who underwent esophagectomy according to the clinical stage (JES 10th)

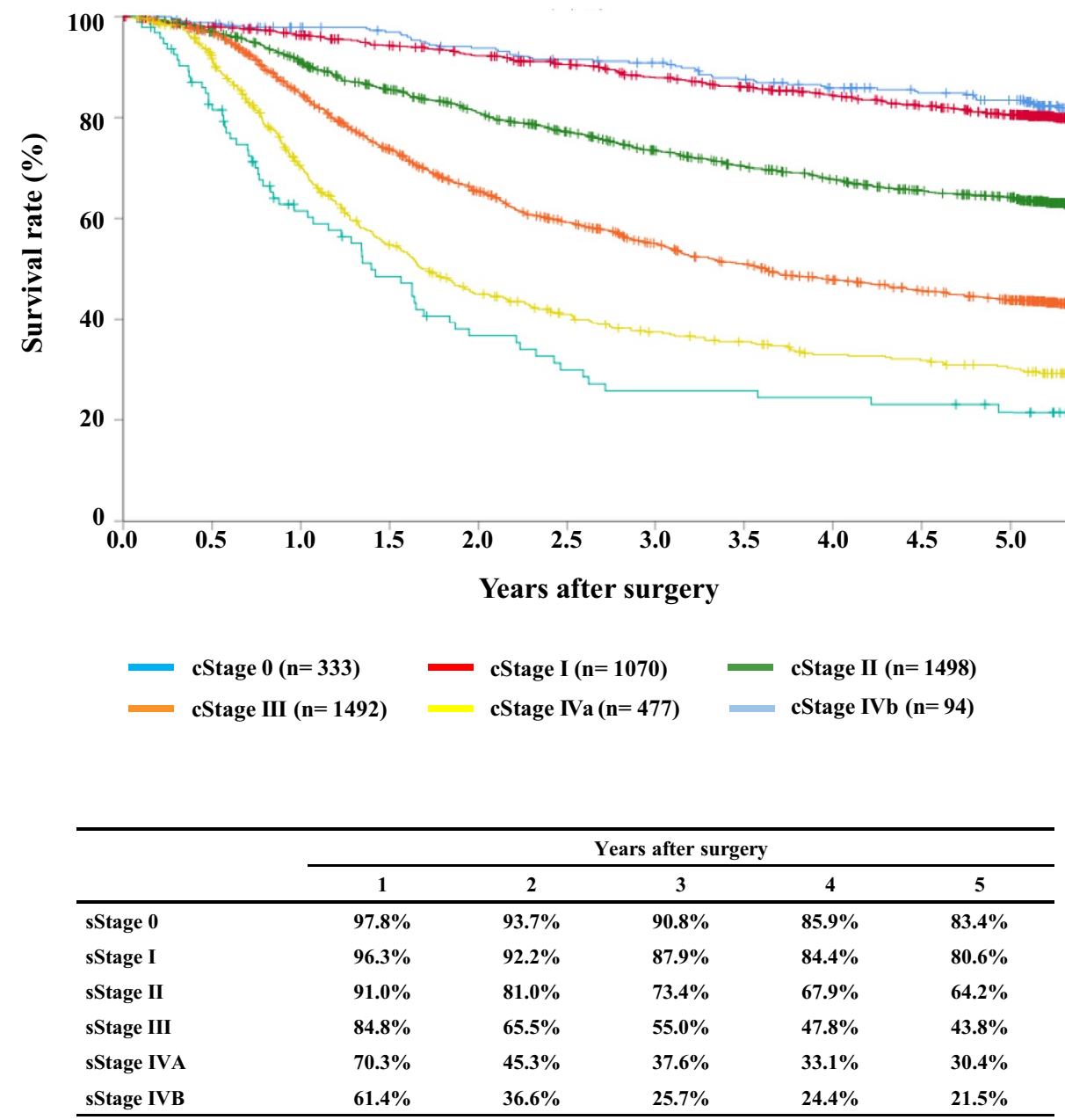


Fig. 9 Survival of patients who underwent esophagectomy according to the clinical stage (UICC TNM 7th)

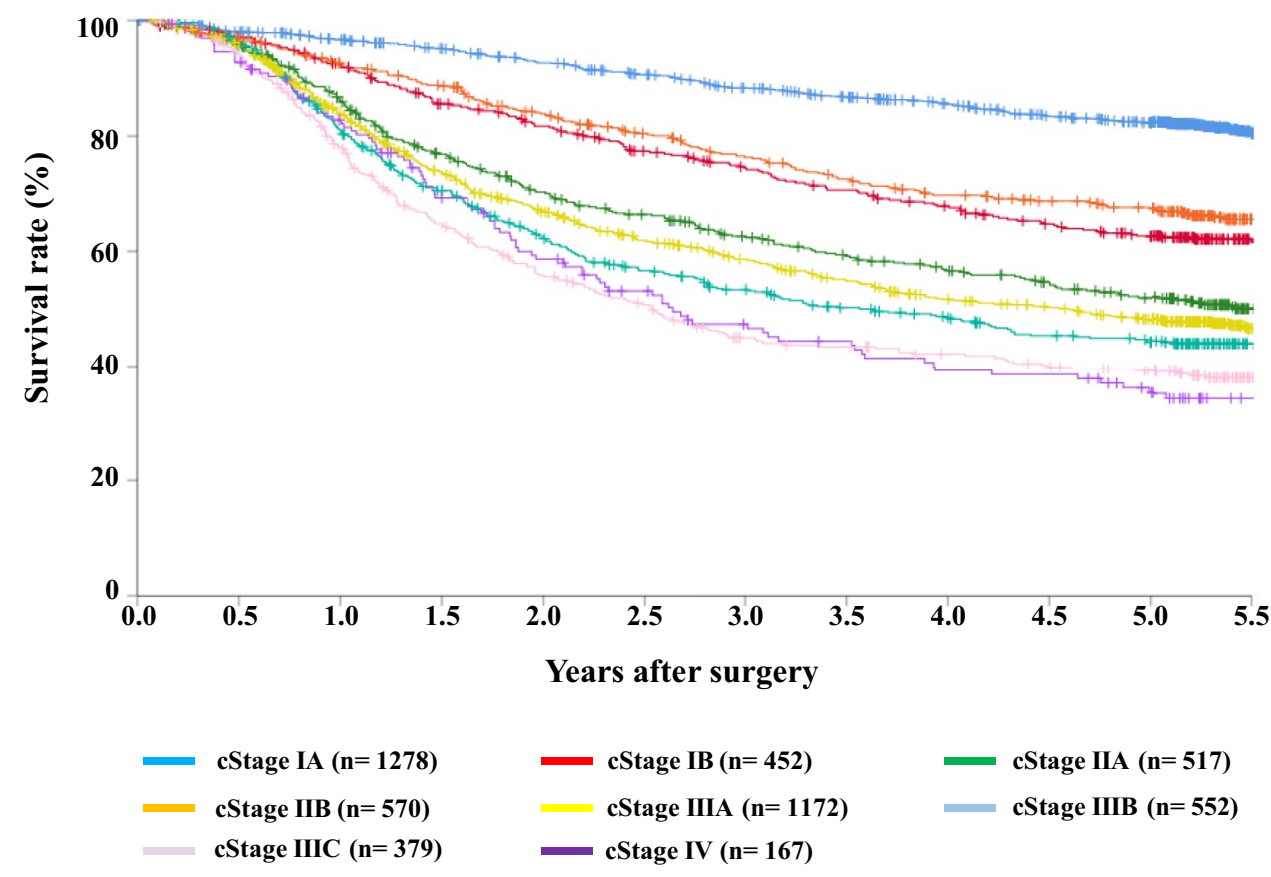

\begin{tabular}{lccccc}
\hline & \multicolumn{5}{c}{ Years after surgery } \\
\cline { 2 - 5 } & 1 & 2 & 3 & 4 & 5 \\
\hline cStage IA & $96.7 \%$ & $92.7 \%$ & $88.2 \%$ & $85.5 \%$ & $82.4 \%$ \\
cStage IB & $91.9 \%$ & $81.8 \%$ & $74.4 \%$ & $67.8 \%$ & $62.6 \%$ \\
cStage IIA & $86.0 \%$ & $70.3 \%$ & $62.4 \%$ & $56.6 \%$ & $52.0 \%$ \\
cStage IIB & $92.5 \%$ & $83.9 \%$ & $76.4 \%$ & $69.8 \%$ & $67.5 \%$ \\
cStage IIIA & $83.8 \%$ & $66.8 \%$ & $58.7 \%$ & $51.7 \%$ & $48.1 \%$ \\
cStage IIIB & $80.8 \%$ & $62.7 \%$ & $53.4 \%$ & $48.5 \%$ & $44.3 \%$ \\
cStage IIIC & $78.1 \%$ & $56.0 \%$ & $45.1 \%$ & $42.2 \%$ & $39.1 \%$ \\
cStage IV & $82.8 \%$ & $58.9 \%$ & $47.7 \%$ & $39.4 \%$ & $35.4 \%$ \\
\hline
\end{tabular}


Fig. 10 Survival of patients who underwent esophagectomy according to the depth of tumor invasion, pT (JES 10th)

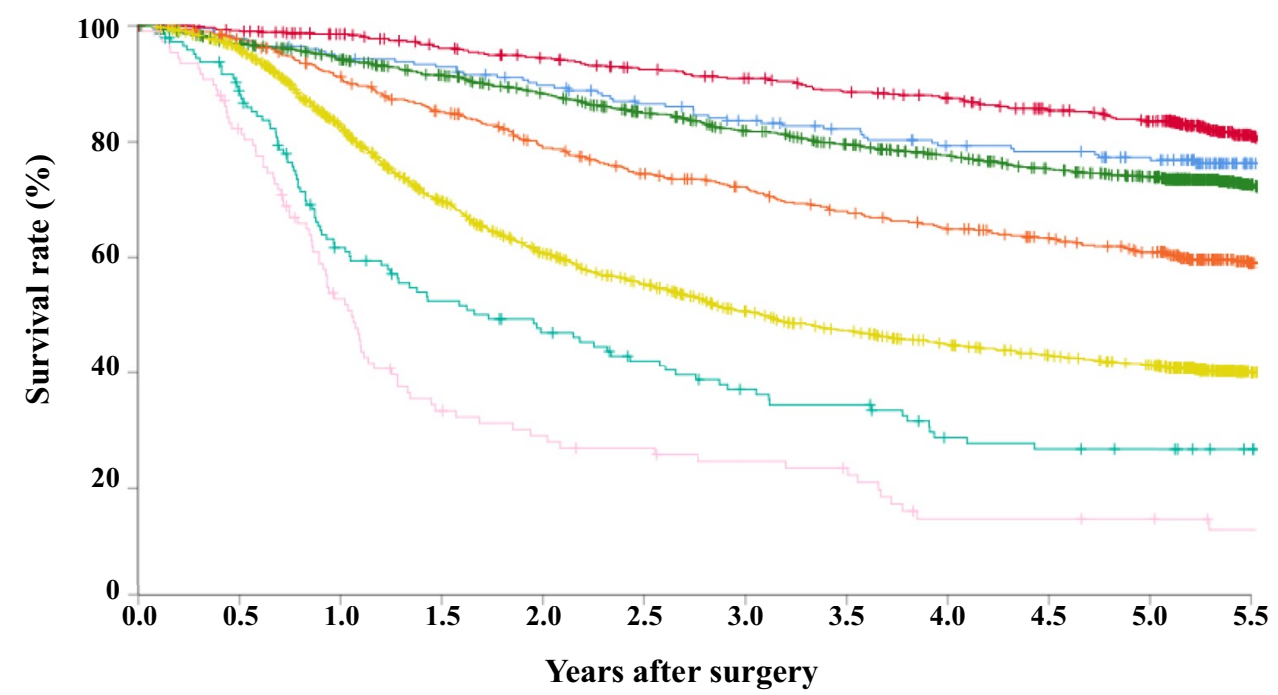

$\begin{array}{lll}\text { pT0 }(n=227) & \text { pT1a }(n=639) & \text { pT1b }(n=1434) \\ \text { pT2 }(n=580) & \text { pT3 }(n=1910) & \text { pT4a }(n=145) \\ \text { pT4b }(n=109) & & \end{array}$

\begin{tabular}{lccccc}
\hline & \multicolumn{5}{c}{ Years after Esophagectomy } \\
\cline { 2 - 5 } & 1 & 2 & 3 & 4 & 5 \\
\hline pT0 & $94.2 \%$ & $89.7 \%$ & $83.7 \%$ & $79.4 \%$ & $76.8 \%$ \\
pT1a & $98.6 \%$ & $94.5 \%$ & $90.9 \%$ & $87.5 \%$ & $83.6 \%$ \\
pT1b & $94.1 \%$ & $88.2 \%$ & $\mathbf{8 1 . 8} \%$ & $77.5 \%$ & $73.8 \%$ \\
pT2 & $90.7 \%$ & $79.0 \%$ & $71.9 \%$ & $65.0 \%$ & $60.9 \%$ \\
pT3 & $82.5 \%$ & $60.8 \%$ & $50.8 \%$ & $45.0 \%$ & $41.3 \%$ \\
pT4a & $62.1 \%$ & $47.4 \%$ & $37.3 \%$ & $28.9 \%$ & $26.9 \%$ \\
pT4b & $53.3 \%$ & $29.3 \%$ & $24.8 \%$ & $14.9 \%$ & $14.9 \%$ \\
\hline
\end{tabular}


Fig. 11 Survival of patients who underwent esophagectomy according to lymph-node metastasis (JES 10th)

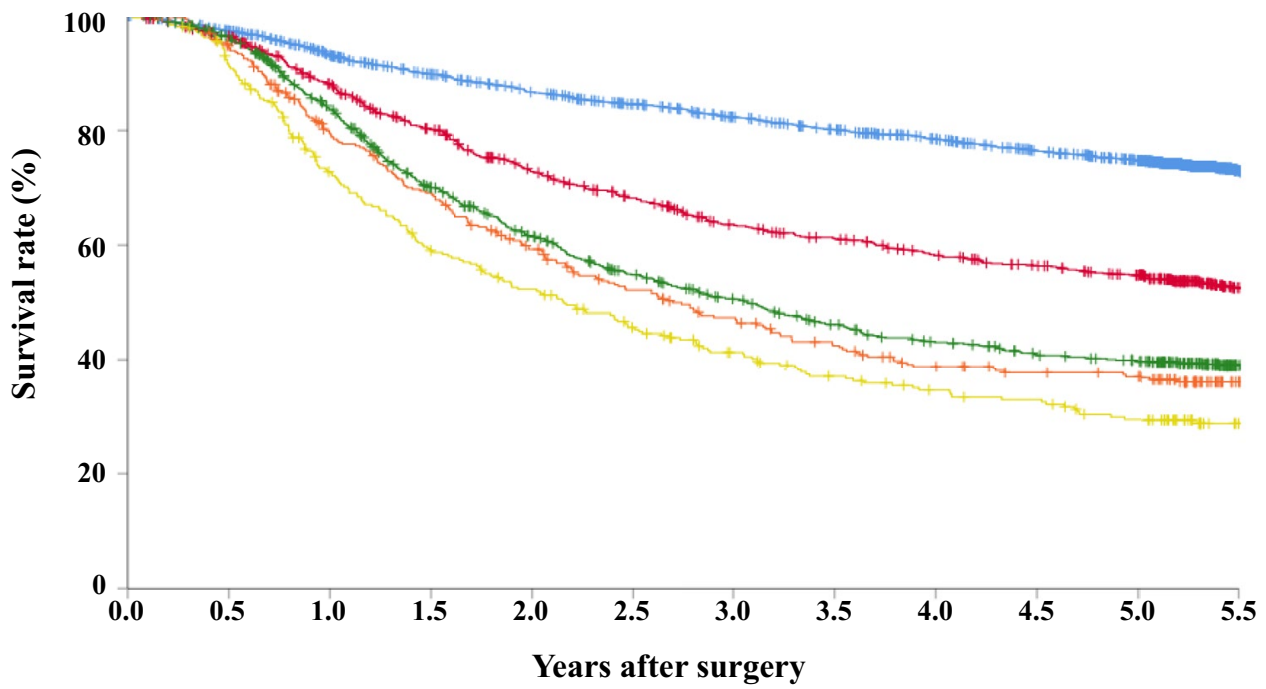

$\begin{array}{ll}\text { pN0 }(n=2525) & \text { pN1 }(n=942) \\ \text { pN3 }(n=366) & \text { pN4 }(n=316)\end{array}$

\begin{tabular}{|c|c|c|c|c|c|}
\hline & \multicolumn{5}{|c|}{ Years after Esophagectomy } \\
\hline & 1 & 2 & 3 & 4 & 5 \\
\hline pNo & $93.0 \%$ & $86.7 \%$ & $82.3 \%$ & $78.5 \%$ & $74.7 \%$ \\
\hline pN1 & $88.0 \%$ & $72.9 \%$ & $63.5 \%$ & $58.2 \%$ & $54.8 \%$ \\
\hline pN2 & $83.9 \%$ & $61.7 \%$ & $50.6 \%$ & $43.1 \%$ & $39.7 \%$ \\
\hline pN3 & $79.4 \%$ & $59.2 \%$ & $47.4 \%$ & $38.7 \%$ & $36.9 \%$ \\
\hline pN4 & $72.8 \%$ & $52.3 \%$ & $41.2 \%$ & $34.4 \%$ & $29.3 \%$ \\
\hline
\end{tabular}


Fig. 12 Survival of patients who underwent esophagectomy according to lymph-node metastasis (UICC TNM 7th)

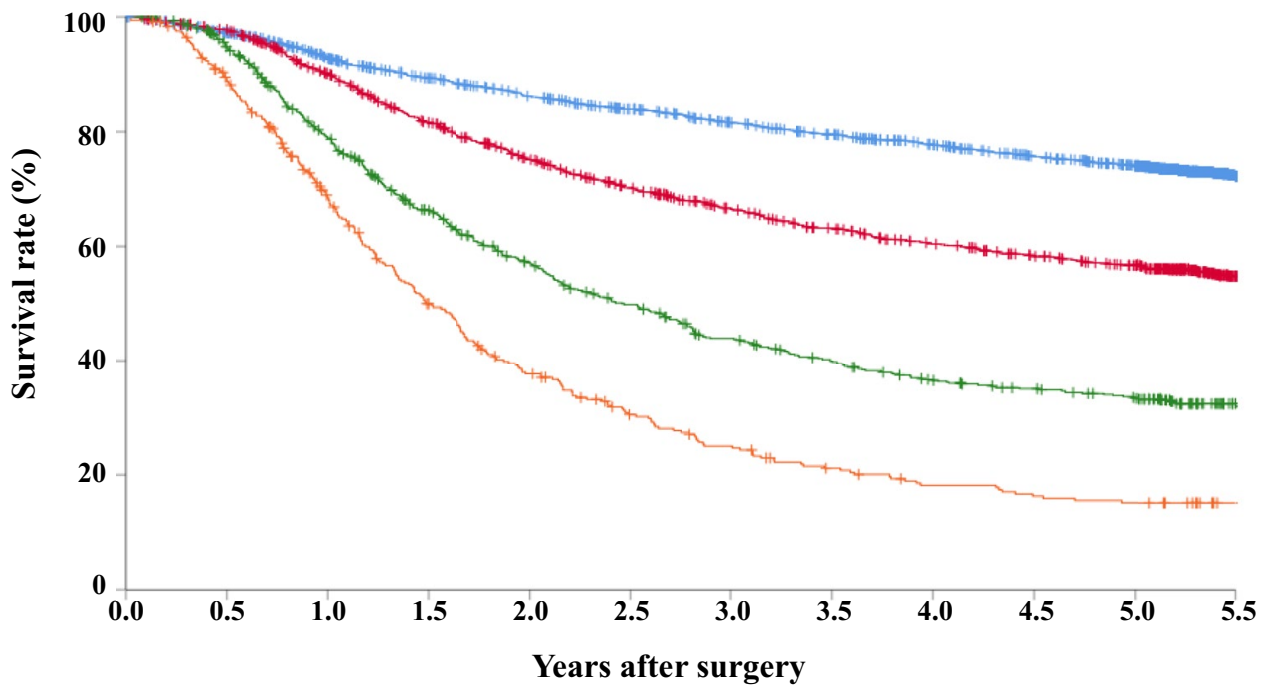

pN0 $(n=2567) \longrightarrow$ pN1 $(n=1369)-\operatorname{pN2}(n=775) \quad$ pN3 $(n=367)$

\begin{tabular}{lccccc}
\hline & \multicolumn{5}{c}{ Years after Esophagectomy } \\
\cline { 2 - 5 } & 1 & 2 & 3 & 4 & 5 \\
\hline pN0 & $92.7 \%$ & $86.2 \%$ & $81.6 \%$ & $77.7 \%$ & $74.0 \%$ \\
pN1 & $90.0 \%$ & $75.1 \%$ & $66.5 \%$ & $60.4 \%$ & $56.6 \%$ \\
pN2 & $79.2 \%$ & $57.3 \%$ & $44.1 \%$ & $36.6 \%$ & $33.4 \%$ \\
pN3 & $68.4 \%$ & $37.8 \%$ & $25.3 \%$ & $18.5 \%$ & $15.4 \%$ \\
\hline
\end{tabular}


Fig. 13 Survival of patients who underwent esophagectomy according to the pathological stage (JES 10th)

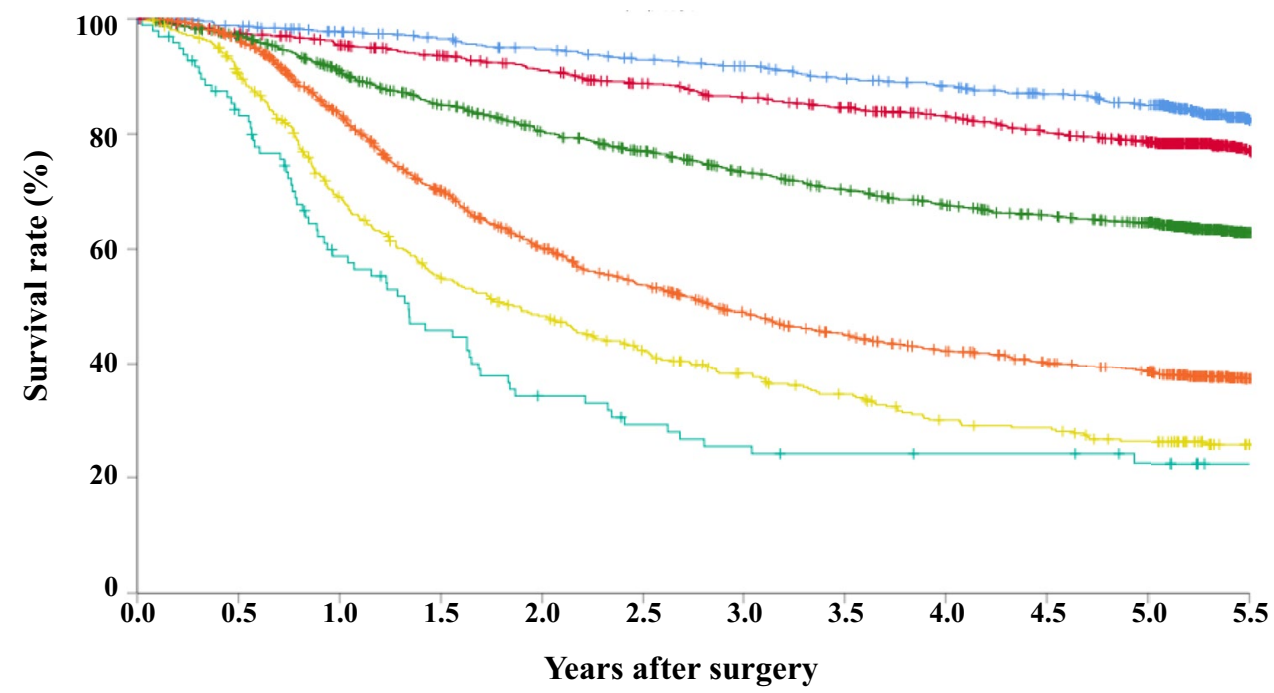

Years after surgery

$\begin{array}{lll}\text { pStage } 0(n=698) & \text { pStage I }(n=942) & \text { pStage II }(n=1440) \\ \text { pStage III }(n=1415) & \text { pStage IVa }(n=418) & \text { pStage } \operatorname{IVb}(n=97)\end{array}$

\begin{tabular}{|c|c|c|c|c|c|}
\hline & \multicolumn{5}{|c|}{ Years after Esophagectomy } \\
\hline & 1 & 2 & 3 & 4 & 5 \\
\hline pStage 0 & $97.7 \%$ & $94.7 \%$ & $91.8 \%$ & $88.4 \%$ & $85.0 \%$ \\
\hline pStage I & $95.4 \%$ & $91.1 \%$ & $86.3 \%$ & $83.1 \%$ & $78.5 \%$ \\
\hline pStage II & $90.7 \%$ & $80.5 \%$ & $73.3 \%$ & $67.7 \%$ & $64.6 \%$ \\
\hline pStage III & $83.4 \%$ & $60.3 \%$ & $49.1 \%$ & $42.3 \%$ & $38.5 \%$ \\
\hline pStage IVA & $69.3 \%$ & $48.5 \%$ & $38.1 \%$ & $30.0 \%$ & $26.3 \%$ \\
\hline pStage IVB & $59.1 \%$ & $34.3 \%$ & $25.6 \%$ & $24.2 \%$ & $22.7 \%$ \\
\hline
\end{tabular}


Fig. 14 Survival of patients who underwent esophagectomy according to the pathological stage (UICC TNM 7th)

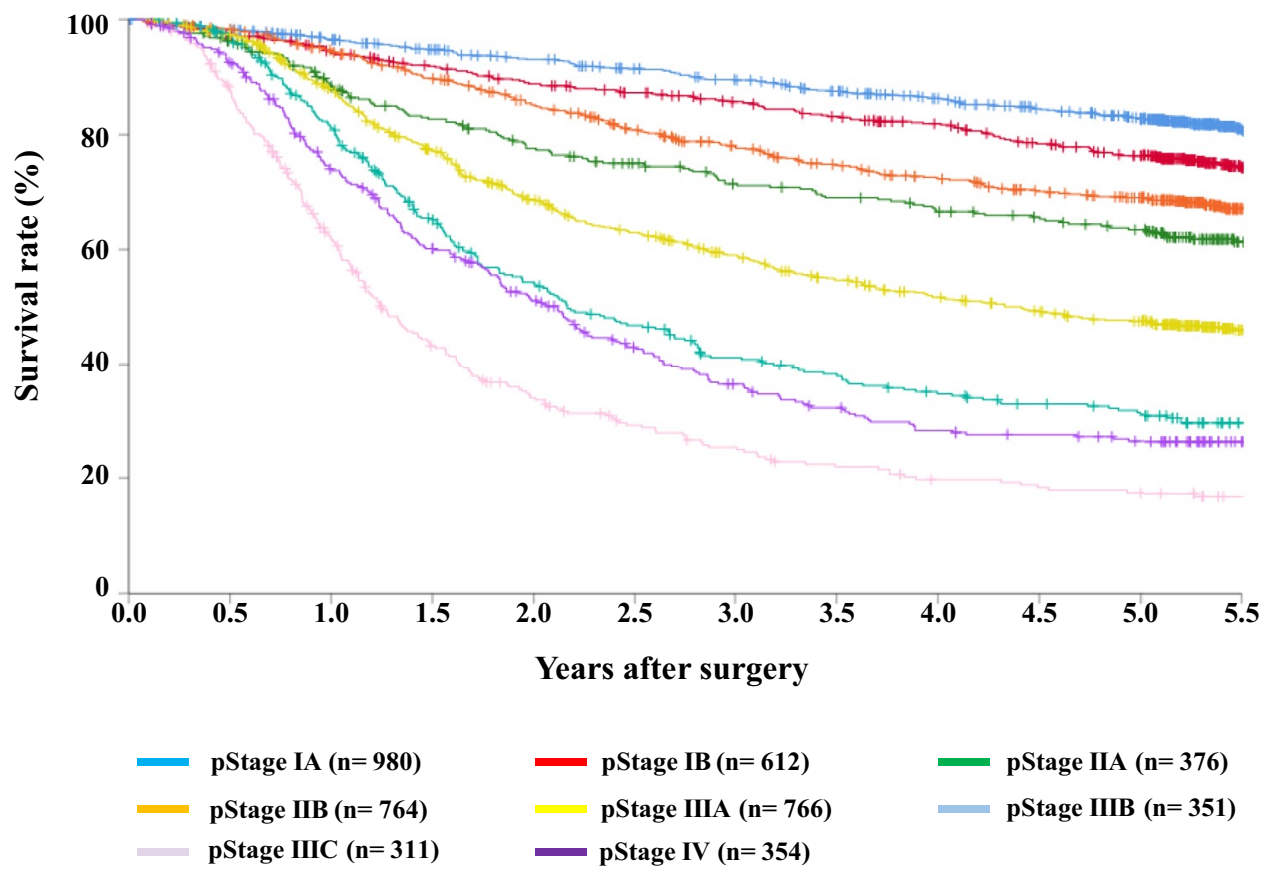

\begin{tabular}{lccccc}
\hline & \multicolumn{5}{c}{ Years after Esophagectomy } \\
\cline { 2 - 5 } & 1 & 2 & 3 & 4 & 5 \\
\hline pStage IA & $96.5 \%$ & $93.1 \%$ & $89.4 \%$ & $86.3 \%$ & $82.7 \%$ \\
pStage IB & $94.1 \%$ & $88.8 \%$ & $85.7 \%$ & $81.9 \%$ & $76.3 \%$ \\
pStage IIA & $88.1 \%$ & $77.6 \%$ & $71.5 \%$ & $66.6 \%$ & $63.5 \%$ \\
pStage IIB & $94.4 \%$ & $85.2 \%$ & $77.6 \%$ & $72.4 \%$ & $69.0 \%$ \\
pStage IIIA & $87.8 \%$ & $68.6 \%$ & $59.0 \%$ & $51.7 \%$ & $47.5 \%$ \\
pStage IIIB & $81.3 \%$ & $54.7 \%$ & $41.5 \%$ & $34.9 \%$ & $31.2 \%$ \\
pStage IIIC & $61.7 \%$ & $34.1 \%$ & $25.6 \%$ & $19.9 \%$ & $17.5 \%$ \\
pStage IV & $74.2 \%$ & $51.3 \%$ & $36.5 \%$ & $28.5 \%$ & $26.6 \%$ \\
\hline
\end{tabular}


Fig. 15 Survival of patients

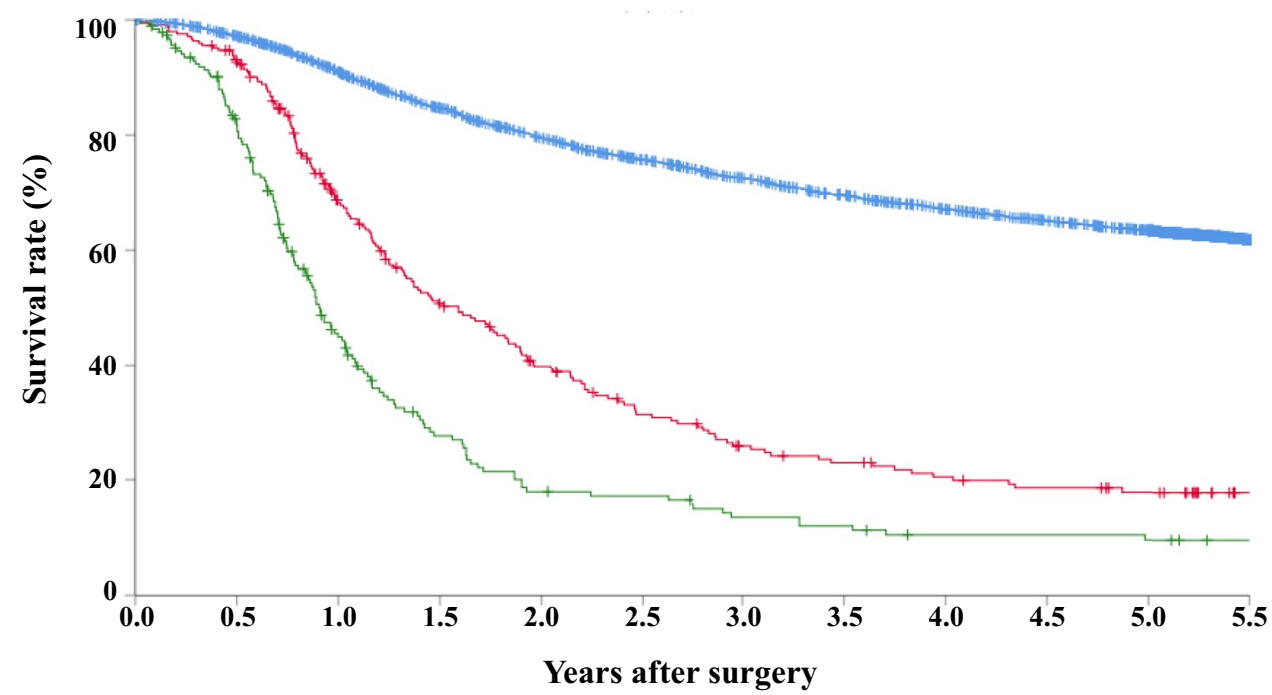

R0 $(n=4585) \quad R 1(n=246) \quad R 2(n=186)$

\begin{tabular}{lccccc}
\hline & \multicolumn{5}{c}{ Years after Esophagectomy } \\
\cline { 2 - 5 } & 1 & 2 & 3 & 4 & 5 \\
\hline R0 & $91.0 \%$ & $79.5 \%$ & $72.5 \%$ & $67.2 \%$ & $63.4 \%$ \\
R1 & $68.2 \%$ & $39.8 \%$ & $25.9 \%$ & $20.5 \%$ & $17.9 \%$ \\
R2 & $46.0 \%$ & $19.1 \%$ & $14.6 \%$ & $11.1 \%$ & $10.2 \%$ \\
\hline
\end{tabular}

\section{Declarations}

Ethical statement All procedures followed in accordance with the ethical standards of the responsible committee on human experimentation (institutional and national) and with the Helsinki Declaration of 1964 and later versions.

Conflict of interest Shiyori Usune, Arata Takahashi, and Hiroaki Miyata are affiliated with the Department of Healthcare Quality Assessment at the University of Tokyo. The department is a social collaboration department supported by grants from the National Clinical Database, Johnson \& Johnson K.K., and Nipro Co. Other authors have no conflict of interest.

Open Access This article is licensed under a Creative Commons Attribution 4.0 International License, which permits use, sharing, adaptation, distribution and reproduction in any medium or format, as long as you give appropriate credit to the original author(s) and the source, provide a link to the Creative Commons licence, and indicate if changes were made. The images or other third party material in this article are included in the article's Creative Commons licence, unless indicated otherwise in a credit line to the material. If material is not included in the article's Creative Commons licence and your intended use is not permitted by statutory regulation or exceeds the permitted use, you will need to obtain permission directly from the copyright holder. To view a copy of this licence, visit http://creativecommons.org/licenses/by/4.0/.

\section{References}

1. Japan Esophageal Society. Japanese classification of esophageal cancer, 10th edition: part 1. Esophagus. 2009;6:1-25.

2. Sobin LH, Gospodarowicz MK, Wittekind C. UICC International Union Against Cancer TNM classification of malignant tumors. 7th ed. New York: Wiley-Blackwell; 2009.

Publisher's Note Springer Nature remains neutral with regard to jurisdictional claims in published maps and institutional affiliations. 


\section{Authors and Affiliations}

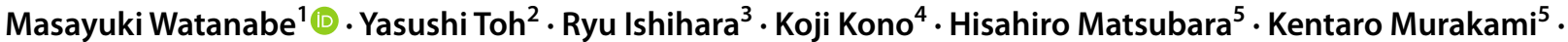

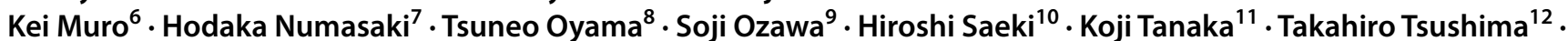 Masaki Ueno ${ }^{13}$. Takashi Uno ${ }^{14} \cdot$ Toshiyuki Yoshio $^{15}$. Shiyori Usune ${ }^{16} \cdot$ Arata Takahashi $^{16} \cdot$ Hiroaki Miyata $^{16} \cdot$ The Registration Committee for Esophageal Cancer of the Japan Esophageal Society}

1 Department of Gastroenterological Surgery, Cancer Institute Hospital of Japanese Foundation for Cancer Research, 3-8-31 Ariake, Koto-ku, Tokyo 135-8550, Japan

2 Department of Gastroenterological Surgery, National Hospital Organization Kyushu Cancer Center, 3-1-1 Notame, Minami-ku, Fukuoka 811-1395, Japan

3 Department of Gastrointestinal Oncology, Osaka International Cancer Institute, 3-1-69 Otemae, Chuo-ku, Osaka 541-8567, Japan

4 Department of Gastrointestinal Tract Surgery, Fukushima Medical University School of Medicine, 1 Hikarigaoka, Fukushima 960-1295, Japan

5 Department of Frontier Surgery, Graduate School of Medicine, Chiba University, 1-8-1 Inohana, Chuo-ku, Chiba 260-8670, Japan

6 Department of Clinical Oncology, Aichi Cancer Center Hospital, 1-1 Kanokoden, Chikusa-ku, Nagoya 464-8681, Japan

7 Department of Medical Physics and Engineering, Graduate School of Medicine, Osaka University, 2-2 Yamadaoka, Suita 565-0871, Japan

8 Department of Endoscopy, Saku Central Hospital Advanced Care Center, 3400-28 Nakagomi, Saku 385-0051, Japan
9 Department of Gastroenterological Surgery, Tokai University School of Medicine, 143 Shimokasuya, Isehara 259-1193, Japan

10 Department of General Surgical Science, Graduate School of Medicine, Gunma University, 3-39-22 Showa-machi, Maebashi 371-8511, Japan

11 Department Gastroenterological Surgery, Graduate School of Medicine, Osaka University, 2-2 Yamadaoka, Suita 565-0871, Japan

12 Division of Gastroenterological Oncology, Shizuoka Cancer Center, 1007 Shimonagakubo, Nagaizumi-cho, Sunto-gun, Shizuoka 411-8777, Japan

13 Department of Gastroenterological Surgery, Toranomon Hospital, 2-2-2 Toranomon, Minato-ku, Tokyo 105-8470, Japan

14 Department of Diagnostic Radiology and Radiation Oncology, Graduate School of Medicine, Chiba University, 1-8-1 Inohana, Chuo-ku, Chiba 260-8670, Japan

15 Department of Upper Gastrointestinal Medicine, Cancer Institute Hospital of Japanese Foundation for Cancer Research, 3-8-31 Ariake, Koto-ku, Tokyo 135-8550, Japan

16 Department of Healthcare Quality Assessment, Graduate School of Medicine, The University of Tokyo, 7-3-1 Hongo, Bunkyo-ku, Tokyo 113-8655, Japan 